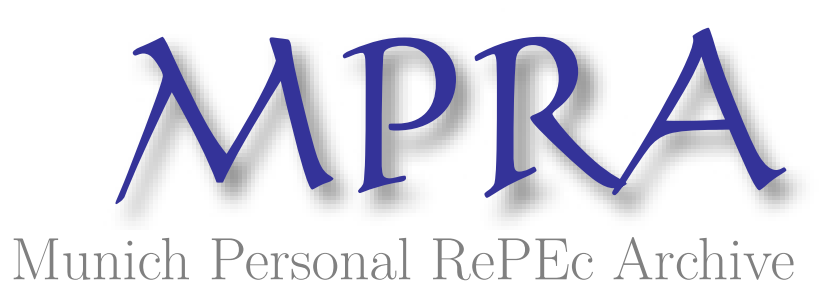

Models of Continuous Dynamics on the
2-Simplex and Applications in Economics

Stijepic, Denis

University of Hagen

23 April 2018

Online at https://mpra.ub.uni-muenchen.de/86341/

MPRA Paper No. 86341, posted 23 Apr 2018 15:38 UTC 


\title{
Models of Continuous Dynamics on the 2-Simplex and Applications in Economics
}

\author{
Denis Stijepic ${ }^{1}$ \\ 1. University of Hagen (Fernuniversität in Hagen), Lehrstuhl für Makroökonomik, Universitätsstraße 41, D-58084 Hagen, Germany.
}

$23^{\text {rd }}$ April 2018

\begin{abstract}
In this paper, we discuss the models of continuous dynamics on the 2-simplex that arise when different qualitative restrictions are imposed on the (continuous) functions that generate the dynamics on the 2-simplex. We consider three types of qualitative restrictions: inequality (or set-theoretical) conditions, monotonicity/curvature (or differential-geometrical) conditions, and topological conditions (referring to (transversal) non-(self-)intersection of trajectories). We discuss the implications of these restrictions for transitional and limit dynamics on the 2-simplex and the wide range of potential and existing applications of the resulting system-theoretical models in economics and, in particular, in economic growth and development theory.
\end{abstract}

Key words: dynamics, trajectory, 2-simplex, continuous, monotonous, intersection, self-intersection, Poincaré-Bendixson, economics

\section{Introduction}

In this paper, we discuss the models of continuous dynamics on the 2-simplex that arise when different qualitative restrictions are imposed on the (continuous) vector function $\mathbf{x}(t) \equiv\left(x_{1}(t), x_{2}(t), x_{3}(t)\right)$ that generates the dynamics on the 2-simplex (where $t$ represents time). In particular, there are three major types of qualitative conditions that can be imposed on this function:

(1.) inequality conditions of the type $\forall t \in A \forall i \in B$ $x_{i}(t) \lessgtr a_{i}=$ const., which can be treated by using set-theoretical concepts (referring to the points or segments of the corresponding trajectory and the partitions of the 2-simplex);

(2.) (strict) monotonicity conditions referring to all or some of the functions $x_{i}(t)$, which can be treated by (differential) geometrical concepts of tangential vector angles and curvature; and

Corresponding author: Dr. Denis Stijepic, research field: economics, growth, development, structural change, systems theory. E-mail: denis.stijepic@fernuni-hagen.de.
(3.) conditions regarding (transversal) trajectory non-(self-)inter-sections, which can be treated by using topological concepts (e.g., homeomorphisms).

We discuss the implications of these restrictions for the transitional and limit dynamics on the 2-simplex (among others, fixed points, waves, or (limit) cycles may arise). The models that result from this discussion are relatively simple from the mathematical point of view, yet they seem widely applicable in economic growth and development theory and, thus, may be regarded as powerful system-theoretical constructs. Moreover, although the 2-simplex can be regarded as a bounded subset of a plane (in $\mathbb{R}^{3}$ ), the description of the dynamics on the 2-simplex requires a greater variety of analytical concepts in comparison to the description of the dynamics in $\mathbb{R}^{2}$ (see, e.g., the discussion of the monotonicity concepts in Sections 2.3 and 3) and, thus, merits a detailed consideration.

The rest of the paper is organized as follows. In Section 2, we discuss the characterization of trajectory families on the 2-simplex via geometrical and topological concepts. Sections 3-5 discuss the 
implications of these concepts for transitional and limit dynamics on the 2-simplex. This discussion yields system-theoretical models. The potential and existing applications of these models in economics and, in particular, in growth and development theory are discussed in Section 6. Concluding remarks are provided in Section 7.

\section{Characterization of the Trajectories on the 2-Simplex}

In Section 2, we summarize the concepts that can be used to characterize continuous dynamics on the 2-simplex as applied by Stijepic (2015, 2017a,b) in structural change modeling. While there are different mathematical notational conventions, we choose the following notation for reasons of simplicity: small letters (e.g., $x$ ), bold small letters (e.g., $\mathbf{x}$ ), capital letters (e.g., $X$ ), and Greek letters (e.g., $\alpha$ ) denote scalars, vectors/points, sets, and vector angles, respectively. A dot indicates a derivative with respect to time (e.g., $\dot{x}$ is the derivative of $x$ with respect to time). $\mathbb{R}$ is the set of real numbers, and $\mathbb{N}$ is the set of natural numbers (including zero). $\operatorname{cl}(A)$ denotes the closure of the set $A$. If $I$ denotes an open interval (e.g., $(a, b)$ ), then [I], [I), and (I] denote the corresponding closed (e.g., $[a, b])$, left-closed (e.g., $[a, b)$ ), and right-closed (e.g., $(a, b])$ interval, respectively.

\subsection{Trajectories on the 2-Simplex}

The (standard) 2-simplex $(S)$, which is defined by (1), is a triangle in $\mathbb{R}^{3}$, as depicted by Figure 1 . The Cartesian coordinates of the simplex vertices $\mathbf{v}_{1}, \mathbf{v}_{2}$, and $\mathbf{v}_{3}$ are stated by (2).
(1) $S:=\left\{\left(x_{1}, x_{2}, x_{3}\right) \in \mathbb{R}^{3}: x_{1}+x_{2}+x_{3}=1 \wedge \forall i \in\{1,2\right.$, 3\} $\left.0 \leq x_{i} \leq 1\right\}$
(2a) $\mathbf{v}_{1}:=(1,0,0)$
(2b) $\mathbf{v}_{2}:=(0,1,0)$
(2c) $\mathbf{v}_{3}:=(0,0,1)$

We define the vector function $\mathbf{x}(t, \mathbf{j})$ as follows:

(3a) $\mathbf{x}(t, \mathbf{j}) \equiv\left(x_{1}(t, \mathbf{j}), x_{2}(t, \mathbf{j}), x_{3}(t, \mathbf{j})\right): T \times J \rightarrow S$

(3b) $0 \in T \subseteq \mathbb{R}$ (3c) $J \subseteq S$.

The trajectory $X(T, \mathbf{j})$ and the trajectory segment $X\left(T^{+}, \mathbf{j}\right)$ are defined by (4).

(4a) $\forall \mathbf{j} \in J \quad X(T, \mathbf{j}):=\{\mathbf{x}(t, \mathbf{j}) \in S: t \in T\}$

(4b) $\forall \mathbf{j} \in J \quad X\left(T^{+}, \mathbf{j}\right):=\{t \in T: t \geq 0\}$

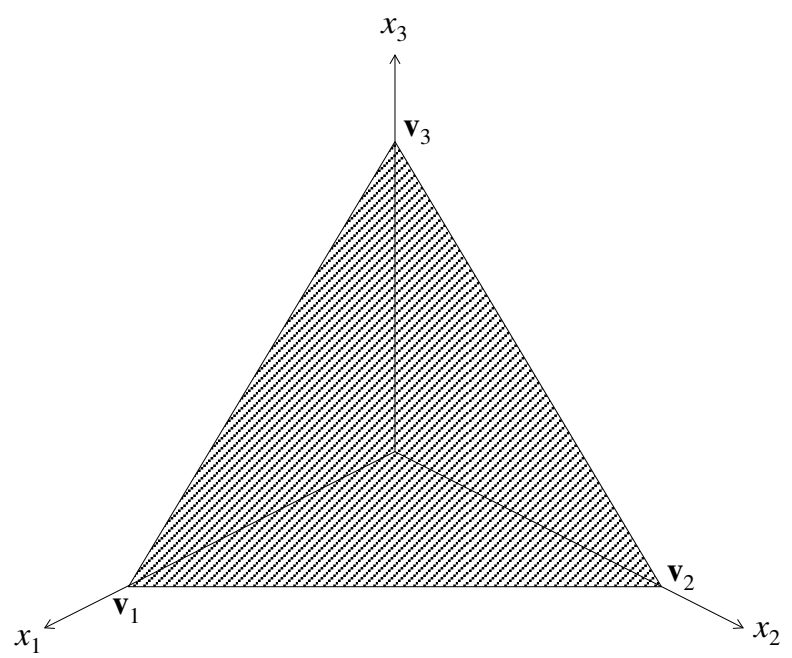

Figure 1. The standard simplex $S$ in $\mathbb{R}^{3}$.

In fact, (4a) defines a trajectory family indexed by the set $J$, where each trajectory $X(T, \mathbf{j})$ describes a path on $S$ that is traversed over the period $T . X\left(T^{+}, \mathbf{j}\right)$ is the segment of this path that is traversed over $t \geq 0$.

\subsection{Set-Theoretical Trajectory Classification}

(5) introduces a partitioning of $S$, which can be used for describing the location of relevant trajectory points or segments (e.g., initial segment/state, empirically observed segment, or segment representing the future dynamics), as we will see later.

(5a) $\forall i \in\{1,2,3\} S_{\mathrm{v} i}:=\left\{\left(x_{1}, x_{2}, x_{3}\right) \in S: x_{i}>1 / 2\right\}$

(5b) $S_{\mathrm{v} 0}:=S \backslash\left(S_{\mathrm{v} 1} \cup S_{\mathrm{v} 2} \cup S_{\mathrm{v} 3}\right)$

(5a) and (1) imply that the partition $S_{\mathrm{v} i}$ contains all the points of $S$ that are dominated by $x_{i}$; i.e., if a point $\left(\underline{x}_{1}, \underline{x}_{2}, \underline{x}_{3}\right)$ is located in partition $S_{\mathrm{v} i}$, then $\forall j \in\{1,2,3\} \backslash i$ $\underline{x}_{i}>\underline{x}_{j}$. The geometrical interpretation of the partitionning (5) is depicted in Figure 2. As we can see, for $i \in$ $\{1,2,3\}$, the partition $S_{\mathrm{v} i}$ contains all the points of $S$ that are closer to the vertex $\mathbf{v}_{i}$ than to the other vertices $\left(\mathbf{v}_{j}, j \neq i\right)$. 


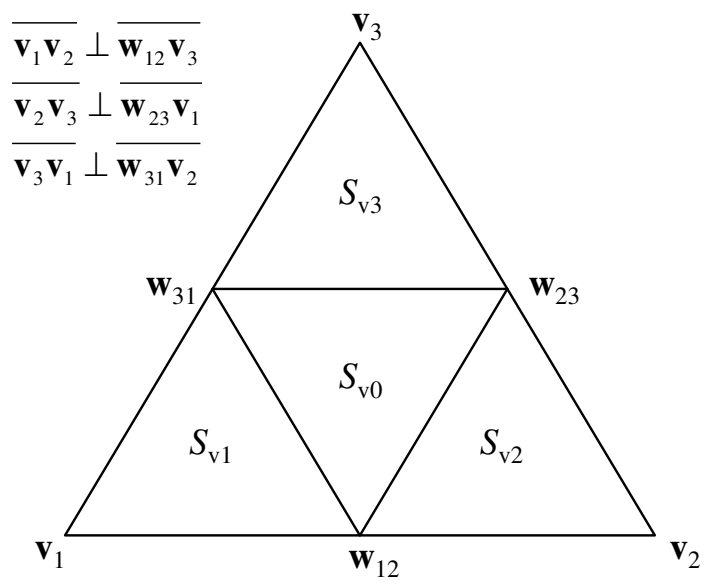

Figure 2. The partitioning of $S$.

The following (set-theoretical) definitions allow us to assess the prediction range of monotonous models, as we will see later. Let $a(K)$ denote the area function assigning the to a set $K \subseteq S$ the (real number indicating the) area of $K . B(T, F):=\bigcup_{\mathbf{j} \in F} X(T, \mathbf{j})$ is the image of the family $F$ of trajectories $X(T, \mathbf{j}), \mathbf{j} \in F \subseteq J$ (cf. (4)). Among all the path-connected and closed subsets of $S$ that cover $B(T, F)$, let $M(T, F)$ denote one of the sets that cover the smallest area of $S . a^{*}(T, F):=a(M(T, F))$ is the family image size of the family $F$.

\subsection{Differential-Geometrical Trajectory Classification}

While the previous discussion can be used for a set-theoretical characterization of trajectories, we focus now on a differential-geometrical characterization of trajectories referring to the angles of the tangential vectors and expressing the monotonicity characteristics and the curvature of a trajectory.

We say that the trajectory $X(T, \mathbf{j})$ is continuous if for the given $\mathbf{j}, \mathbf{x}(t, \mathbf{j})$ is continuous in $t$ on the time interval $T$ (cf. (4a)). Moreover, a trajectory family is continuous if all the trajectories belonging to this family are continuous.

Let (a) $\mathbf{d}(t, \mathbf{j})$ be the directional (or tangential) vector associated with the point $\mathbf{x}(t, \mathbf{j})$, (b) $\ell$ be a line through the point $\mathbf{x}(t, \mathbf{j})$ that is parallel to the simplex edge $\mathbf{v}_{1}-\mathbf{v}_{2}$, and (c) $\delta(t, \mathbf{j}):=\Varangle(\mathbf{d}(t, \mathbf{j}), \ell) \in\left[0^{\circ}, 360^{\circ}\right]$ be the angle between the directional vector $\mathbf{d}(t, \mathbf{j})$ and the line $\ell$ (cf. Figure 3 ).

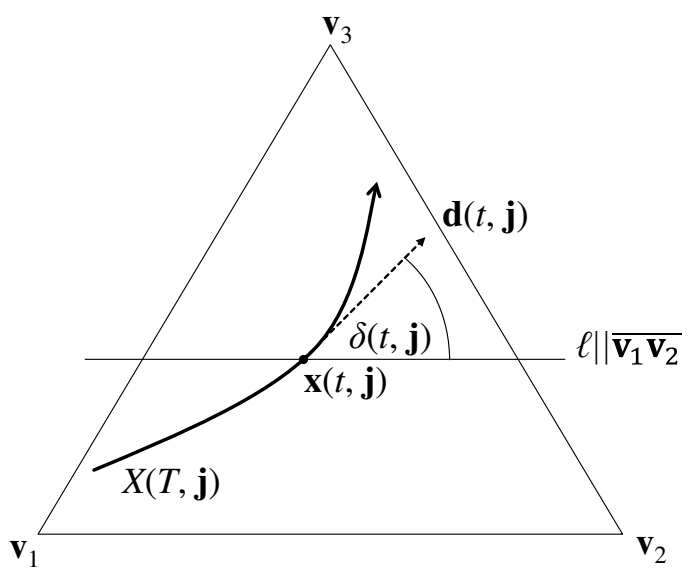

Figure 3. The vector angle $\delta(t, \mathbf{j})$.

Moreover, we define the angles $\alpha_{i}$ and the angle intervals $I_{i}$ by relying on the 'saw tooth' function $\phi: \mathbb{R}$ $\rightarrow \mathbb{R}$ as follows (cf. Figure 4):

(6a) $\phi(z)=[(z-1) / 6-$ floor $((z-1) / 6)] 360^{\circ}$

(6b) $\forall i \in \mathbb{N} \quad I_{i} \equiv\left(\alpha_{i}, \alpha_{i+1}\right):=(\phi(i), \phi(i+1))$

(6c) $\forall i \in \mathbb{N} \forall j \in\{n \in \mathbb{N}: i<n\} \quad\left[I_{i \sim j}\right]:=\bigcup^{j}{ }_{k=i}\left[I_{k}\right] \wedge$ $\left[I_{i \sim j}\right):=\bigcup^{j}{ }_{k=i}\left[I_{k}\right] \backslash \alpha_{j+1} \wedge\left(I_{i \sim j}\right]:=\bigcup^{j}{ }_{k=i}\left[I_{k}\right] \backslash \alpha_{i}$ $\wedge I_{i \sim j}:=\bigcup^{j}{ }_{k=i}\left[I_{k}\right] \backslash \alpha_{i} \backslash \alpha_{j+1}$

By using our definition of the vector angle $\delta(t, \mathbf{j})$ and the vector angles and intervals (6), we can formulate the Properties 1-3 reflecting the relation between the tangential vector angles and the dynamics of $\mathbf{x}(t, \mathbf{j})$ in the case that $\dot{\mathbf{x}}(t, \mathbf{j}) \neq 0$ (cf. Figures 1,3 , and 4).

Property 1. If $\dot{\mathbf{x}}(t, \mathbf{j}) \neq 0$, then (a) $\delta(t, \mathbf{j}) \in I_{3 \sim 5} \Leftrightarrow$ $\dot{x}_{1}(t, \mathbf{j})>0$, (b) $\delta(t, \mathbf{j}) \in I_{0 \sim 2} \Leftrightarrow \dot{x}_{1}(t, \mathbf{j})<0$, and (c) $\delta(t, \mathbf{j})$ $\in\left\{\alpha_{3}, \alpha_{6}\right\} \Leftrightarrow \dot{x}_{1}(t, \mathbf{j})=0$.

Property 2. If $\dot{\mathbf{x}}(t, \mathbf{j}) \neq 0$, then (a) $\delta(t, \mathbf{j}) \in I_{5 \sim 7} \Leftrightarrow$ $\dot{x}_{2}(t, \mathbf{j})>0,(\mathbf{b}) \delta(t, \mathbf{j}) \in I_{2 \sim 4} \Leftrightarrow \dot{x}_{2}(t, \mathbf{j})<0$, and (c) $\delta(t, \mathbf{j})$ $\in\left\{\alpha_{2}, \alpha_{5}\right\} \Leftrightarrow \dot{x}_{2}(t, \mathbf{j})=0$.

Property 3. If $\dot{\mathbf{x}}(t, \mathbf{j}) \neq 0$, then (a) $\delta(t, \mathbf{j}) \in I_{1 \sim 3} \Leftrightarrow$ $\dot{x}_{3}(t, \mathbf{j})>0$, (b) $\delta(t, \mathbf{j}) \in I_{4 \sim 6} \Leftrightarrow \dot{x}_{3}(t, \mathbf{j})<0$, and (c) $\delta(t, \mathbf{j})$ $\in\left\{\alpha_{1}, \alpha_{4}\right\} \Leftrightarrow \dot{x}_{3}(t, \mathbf{j})=0$. 

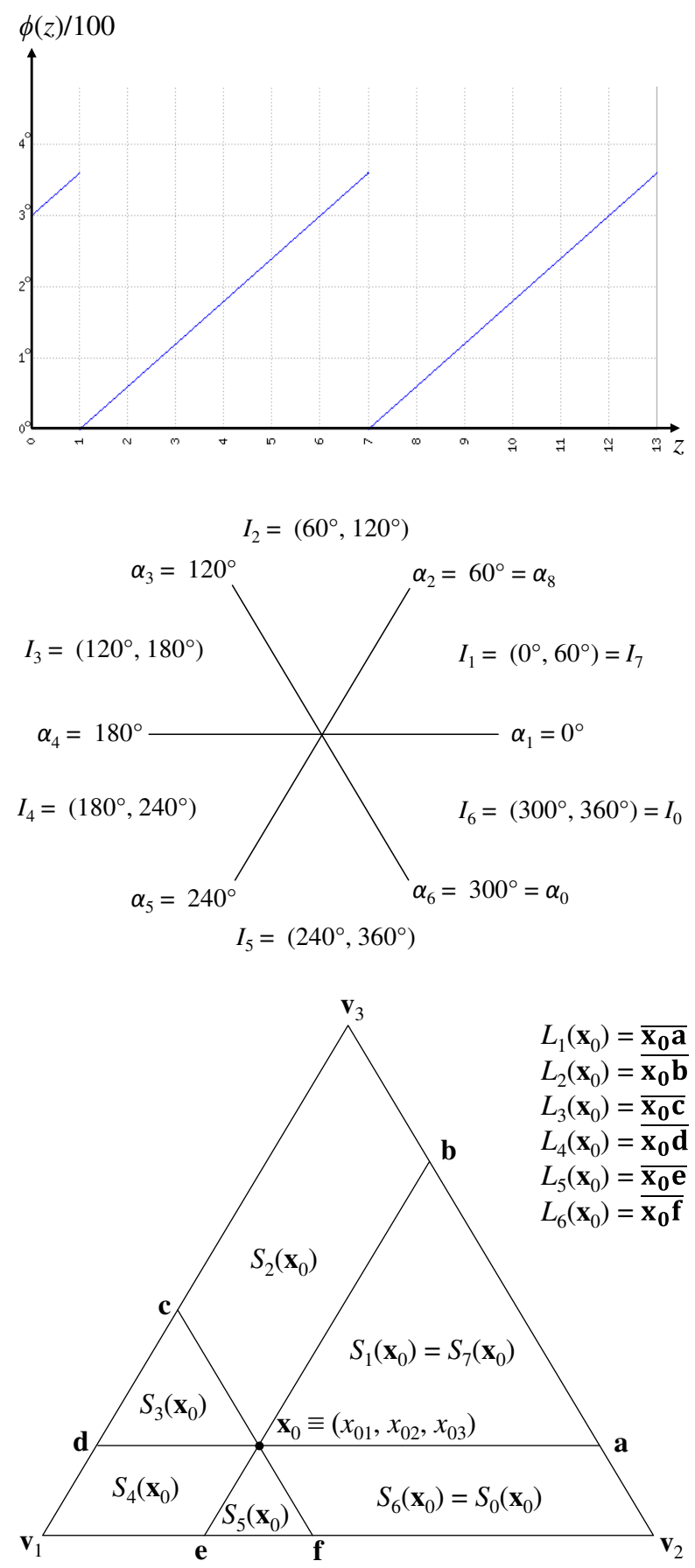

Figure 4. The function $\phi(z)$, the angle intervals $I_{i}$, the line segments $L_{i}\left(\mathbf{x}_{0}\right)$, and the sets $S_{i}\left(\mathbf{x}_{0}\right)$.

We rely on the following definitions of monotonicity. First, $x_{i}(t, \mathbf{j})$ is monotonous (in $\left.t\right)$ if $\left(\forall t \in T \dot{x}_{i}(t, \mathbf{j})\right.$ $\geq 0)$ or $\left(\forall t \in T \dot{x}_{i}(t, \mathbf{j}) \leq 0\right)$. Second, $x_{i}(t, \mathbf{j})$ is strictly monotonous (in $t)$ if either $\left(\forall t \in T \dot{x}_{i}(t, \mathbf{j})>0\right)$ or $(\forall t \in$
$\left.T \dot{x}_{i}(t, \mathbf{j})<0\right)$ but not both. Third, the trajectory $X(T, \mathbf{j})$ (associated with the function $\mathbf{x}(t, \mathbf{j})$ ) is (strictly) monotonous in one dimension if (a) there exists an $i \in\{1,2$, 3 \} such that $x_{i}(t, \mathbf{j})$ is (strictly) monotonous and (b) for all $k \in\{1,2,3\} \backslash i x_{k}(t, \mathbf{j})$ is not (strictly) monotonous. Fourth, the trajectory $X(T, \mathbf{j})$ is (strictly) monotonous in two dimensions if (a) there exist an $i \in\{1,2,3\}$ and a $k \in\{1,2,3\} \backslash i$ such that $x_{i}(t, \mathbf{j})$ and $x_{k}(t, \mathbf{j})$ are (strictly) monotonous and (b) $x_{l}(t, \mathbf{j})$ is not (strictly) monotonous with $l \in\{1,2,3\} \backslash\{i, k\}$. Fifth, the trajectory $X(T, \mathbf{j})$ is (strictly) monotonous (in three dimensions) if $\forall i \in\{1,2,3\} x_{i}(t, \mathbf{j})$ is (strictly) monotonous.

Instead of using the curvature definition that is widespread in differential geometry (and which is difficult to apply in the proofs of our theorems), we use the following definition of curvature relying on vector angles: Let $\beta(q, r, \mathbf{j})$ denote the angle between the two tangential vectors $\mathbf{d}(q, \mathbf{j})$ and $\mathbf{d}(r, \mathbf{j})$ associated with the (monotonous) trajectory $X(T, \mathbf{j})$ on $S$ (cf. (4)), where $q, r \in T$. Among all the tangential vector pairs $(\mathbf{d}(t, \mathbf{j}), \mathbf{d}(s, \mathbf{j}))$ associated with the (monotonous) trajectory $X(T, \mathbf{j})$, where $t, s \in T$, let $\mathbf{d}\left(t^{*}, \mathbf{j}\right)$ and $\mathbf{d}\left(s^{*}, \mathbf{j}\right)$ be among the ones that are characterized by the largest angle $\beta$, i.e., $\beta\left(t^{*}, s^{*}, \mathbf{j}\right)=: \kappa(T, \mathbf{j})$ is maximal tangential vector angle range associated with the trajectory $X(T$, j). The grater $\kappa(T, \mathbf{j})$, the greater the curvature of the trajectory $X(T, \mathbf{j})$. Obviously, a linear trajectory has curvature of 0 . We say that a strictly monotonous trajectory $X(T, \mathbf{j})$ (or a trajectory segment) that describes a clockwise (counterclockwise) movement on $S$ has a positive (negative) signed curvature and write $\underline{\kappa}(T, \mathbf{j})>0(\underline{\kappa}(T, \mathbf{j})<0)$. Let $F$ be a family of trajectories $X(T, \mathbf{j}), \mathbf{j} \in F \subseteq J$ (cf. (4)). Then, $\kappa^{*}(T, F):=$ $\max (\operatorname{cl}(\{\kappa(T, \mathbf{j}): \mathbf{j} \in F\}))$ is the maximum curvature of the family $F$ on the time interval $T$.

\subsection{Topological Trajectory Classification}

Here, the topological characterization refers to the question whether a trajectory family is non-(self-)intersecting, which is a characteristic that can be expressed 
by homeomorphisms. Moreover, it is deciding for the applicability of the Poincare-Bendixson theory (cf. Section 5) that the 2-simplex is homeomorphic to a bounded (and closed) subset of a plane.

Two trajectories $X(T, \mathbf{j})$ and $X(U, \mathbf{k})$ are non-intersecting if $X(T, \mathbf{j}) \cap X(U, \mathbf{k})=\emptyset$, where $U \subseteq \mathbb{R}$. Otherwise they are intersecting. A trajectory $X(T, \mathbf{j})$ is self-intersecting if $\exists(r, s, t) \in T^{3} r<s<t \wedge \mathbf{x}(r, \mathbf{j})=$ $\mathbf{x}(t, \mathbf{j}) \neq \mathbf{x}(s, \mathbf{j})$. Otherwise, the trajectory is non-selfintersecting. According to this definition, a closed trajectory is self-intersecting. A trajectory $X(T, \mathbf{j})$ is transversally self-intersecting if $\exists(t, s) \in T^{2} t \neq s \wedge \mathbf{x}(t$, $\mathbf{j})=\mathbf{x}(s, \mathbf{j}) \wedge \delta(t, \mathbf{j}) \neq \delta(s, \mathbf{j})$. Otherwise, the trajectory is transversally non-self-intersecting. According to this definition, a closed trajectory corresponding to a Jordan curve is transversally non-self-intersecting.

\section{Implications of Monotonicity}

In contrast to monotonous and bounded trajectories in $\mathbb{R}^{2}$, monotonous trajectories on the 2-simplex can have (a) a wide range of different shapes and (b) omega limit sets consisting of more than only one (fixed) point. In this section, we discuss the geometrical aspects of the transitional and limit dynamics associated with continuous trajectories that are monotonous in one, two, or three dimensions. As we will, see these geometrical properties have interesting applications in economic dynamics modeling.

\subsection{General Properties of Monotonous Trajectories on the 2-Simplex}

In this section, we show that continuous trajectories that are monotonous in three dimensions (two dimensions) are characterized by relatively low curvatures, allow for relatively weak waves, and are placed in relatively small subsets of the 2-simplex in comparison to the 'related' trajectories that are monotonous in two dimensions (one dimension). Propositions 1-3 and Corollary 1 summarize these results formally. The readers who are less interested in this formal discussion can also go directly to the discussion of Figure 4 (see the paragraphs below Proposition 3), which elaborates on the intuitive/graphical interpretation of these geometrical properties.

Given a point $\mathbf{x}_{0} \equiv\left(x_{01}, x_{02}, x_{03}\right) \in S$, (7) defines different subsets of $S$. As we will see (in Proposition 3), each of the subsets $S_{1}-S_{6}$ defined by (7) corresponds to the closure of one of the vector angle intervals $I_{1}-I_{6}$ defined by (6) and each of the line segments $L_{1}-L_{6}$ defined by (7) corresponds to one of the angles $\alpha_{1}-\alpha_{6}$ defined by (6) (cf. Figure 4).

(7a) $L_{1}\left(\mathbf{x}_{0}\right):=\left\{\left(x_{1}, x_{2}, x_{3}\right) \in S: x_{1} \leq x_{01} \wedge x_{3}=x_{03}\right\}$

(7b) $S_{1}\left(\mathbf{x}_{0}\right):=\left\{\left(x_{1}, x_{2}, x_{3}\right) \in S: x_{2} \geq x_{02} \wedge x_{3} \geq x_{03}\right\}$

(7c) $L_{2}\left(\mathbf{x}_{0}\right):=\left\{\left(x_{1}, x_{2}, x_{3}\right) \in S: x_{1} \leq x_{01} \wedge x_{2}=x_{02}\right\}$

(7d) $S_{2}\left(\mathbf{x}_{0}\right):=\left\{\left(x_{1}, x_{2}, x_{3}\right) \in S: x_{1} \leq x_{01} \wedge x_{2} \leq x_{02}\right\}$

(7e) $L_{3}\left(\mathbf{x}_{0}\right):=\left\{\left(x_{1}, x_{2}, x_{3}\right) \in S: x_{1}=x_{01} \wedge x_{2} \leq x_{02}\right\}$

(7f) $S_{3}\left(\mathbf{x}_{0}\right):=\left\{\left(x_{1}, x_{2}, x_{3}\right) \in S: x_{1} \geq x_{01} \wedge x_{3} \geq x_{03}\right\}$

(7g) $L_{4}\left(\mathbf{x}_{0}\right):=\left\{\left(x_{1}, x_{2}, x_{3}\right) \in S: x_{1} \geq x_{01} \wedge x_{3}=x_{03}\right\}$

(7h) $S_{4}\left(\mathbf{x}_{0}\right):=\left\{\left(x_{1}, x_{2}, x_{3}\right) \in S: x_{2} \leq x_{02} \wedge x_{3} \leq x_{03}\right\}$

(7i) $L_{5}\left(\mathbf{x}_{0}\right):=\left\{\left(x_{1}, x_{2}, x_{3}\right) \in S: x_{1} \geq x_{01} \wedge x_{2}=x_{02}\right\}$

(7j) $S_{5}\left(\mathbf{x}_{0}\right):=\left\{\left(x_{1}, x_{2}, x_{3}\right) \in S: x_{1} \geq x_{01} \wedge x_{2} \geq x_{02}\right\}$

(7k) $L_{6}\left(\mathbf{x}_{0}\right):=\left\{\left(x_{1}, x_{2}, x_{3}\right) \in S: x_{1}=x_{01} \wedge x_{2} \geq x_{02}\right\}$

(71) $S_{6}\left(\mathbf{x}_{0}\right):=\left\{\left(x_{1}, x_{2}, x_{3}\right) \in S: x_{1} \leq x_{01} \wedge x_{3} \leq x_{03}\right\}$

$(7 \mathrm{~m}) S_{0}\left(\mathbf{x}_{0}\right):=S_{6}\left(\mathbf{x}_{0}\right) \quad S_{7}\left(\mathbf{x}_{0}\right):=S_{1}\left(\mathbf{x}_{0}\right)$

(7n) $\forall i \in \mathbb{N} \forall j \in\{n \in \mathbb{N}: i<n\} S_{i \sim j}\left(\mathbf{x}_{0}\right):=\bigcup j_{k=i} S_{k}\left(\mathbf{x}_{0}\right)$

Proposition 1. Assume that (a) the trajectory $X(T$, j) defined by (4a) is continuous and monotonous in one dimension on $S$, (b) $\mathbf{x}(0, \mathbf{j})=\mathbf{x}_{0} \equiv\left(x_{01}, x_{02}, x_{03}\right) \in S$, and (c) $\forall t \in T \dot{\mathbf{x}}(t, \mathbf{j}) \neq 0$. Then, $X(T, \mathbf{j})$ and $X\left(T^{+}, \mathbf{j}\right)$ (cf. (4b)) satisfy one and only one of the Condition Sets $\mathrm{P} 1_{1}-\mathrm{P} 1_{9}$, which are defined as follows (cf. (6) and (7)):

a) for $i \in\{1,2, \ldots, 6\}$, Condition Set $\mathrm{P} 1_{i}$ is: $(\forall t \in T$ $\left.\delta(t, \mathbf{j}) \in\left[I_{(i-1) \sim(i+1)}\right]\right) \wedge\left(\exists t \in T \delta(t, \mathbf{j}) \in I_{(i-1) \sim(i+1)}\right) \wedge(\exists(r$, $\left.s) \in T^{2} \delta(r, \mathbf{j}) \in\left[I_{i-1}\right) \wedge \delta(s, \mathbf{j}) \in\left(I_{i+1}\right]\right) \wedge X\left(T^{+}, \mathbf{j}\right) \subset$ $S_{(i-1) \sim(i+1)}\left(\mathbf{x}_{0}\right)$;

b) for $i \in\{7,8,9\}$, Condition Set $\mathrm{P} 1_{i}$ is: $(\forall t \in T$ $\left.\delta(t, \mathbf{j}) \in\left\{\alpha_{i-6}, \alpha_{i-3}\right\}\right) \wedge\left(\exists(p, q) \in T^{2} \delta(p, \mathbf{j}) \in\left\{\alpha_{i-6}\right\} \wedge\right.$ $\left.\delta(q, \mathbf{j}) \in\left\{\alpha_{i-6}\right\}\right) \wedge X\left(T^{+}, \mathbf{j}\right) \subseteq L_{i-6}\left(\mathbf{x}_{0}\right) \cup L_{i-3}\left(\mathbf{x}_{0}\right)$.

Proof. As defined in Section $2, X(T, \mathbf{j})$ is monotonous in one dimension if for one and only one $i \in\{1,2$, 
$3\}$, the function $x_{i}(t, \mathbf{j})$ is monotonous while for all other $i, x_{i}(t, \mathbf{j})$ is non-monotonous. Thus, for proving Proposition 1, we have to consider only three alternative scenarios of monotonicity in one dimension: (A) $x_{1}(t, \mathbf{j})$ is monotonous, (B) $x_{2}(t, \mathbf{j})$ is monotonous, and $(\mathrm{C}) x_{3}(t, \mathbf{j})$ is monotonous. Moreover, since a monotonous function can be monotonously increasing or monotonously decreasing (or both), we have three alternative sub-scenarios for each of the three scenarios (A)-(C): (a) monotonously increasing, (b) monotonously decreasing, and (c) both, monotonously increasing and monotonously decreasing (which means constant). Thus, overall, we have nine sub-scenarios: (Aa)-(Ac), (Ba)-(Bc), and (Ca)-(Cc). According to Properties 1-3, each of the Condition Sets $\mathrm{P} 1_{1}-\mathrm{P} 1_{9}$, to which Proposition 1 refers, represents one of the nine sub-scenarios (Aa)-(Cc). For example, Condition Sets $\mathrm{P} 1_{2}, \mathrm{P} 1_{5}$, and $\mathrm{P} 1_{7}$ represent the sub-scenarios $(\mathrm{Ca})$, $(\mathrm{Cb})$, and $(\mathrm{Cc})$, respectively.

Consider first the sub-scenario $(\mathrm{Ca})$, i.e., assume that $x_{3}(t, \mathbf{j})$ increases monotonously. Property 3 and (6b) imply that (8) is valid in sub-scenario $(\mathrm{Ca})$.

(8) $\left(\forall t \in T \delta(t, \mathbf{j}) \in\left[I_{1 \sim 3}\right]\right) \wedge\left(\exists t \in T \delta(t, \mathbf{j}) \in I_{1 \sim 3}\right)$

Moreover, according to the definition of 'monotonicity in one dimension', to which Proposition 1 refers, (9) is valid in sub-scenario (Ca).

(9) $x_{1}(t, \mathbf{j})$ and $x_{2}(t, \mathbf{j})$ are non-monotonous.

The interval $\left[I_{1 \sim 3}\right]$, to which (8) refers, can be partitioned into three subintervals $\left[I_{1}\right),\left[I_{2}\right]$, and $\left(I_{3}\right]$. Properties 1 and 2 and (6b) imply (10).

(10a) $\forall t \in T \delta(t, \mathbf{j}) \in\left(I_{3}\right] \Rightarrow x_{1}(t, \mathbf{j})$ is monotonous.

(10b) $\forall t \in T \delta(t, \mathbf{j}) \in\left[I_{1}\right) \Rightarrow x_{2}(t, \mathbf{j})$ is monotonous.

(10c) $\forall t \in T \delta(t, \mathbf{j}) \in\left[I_{2}\right] \Rightarrow x_{1}(t, \mathbf{j})$ and $x_{2}(t, \mathbf{j})$ are monotonous.

The statements (10) imply statement (11).

(11) $x_{1}(t, \mathbf{j})$ or $x_{2}(t, \mathbf{j})$ is monotonous iffor all $t \in T, \delta(t$, j) is within one and only one of the subintervals $\left[I_{1}\right),\left[I_{2}\right]$, and $\left(I_{3}\right]$.

(9) and (11) imply that over the period $T$, the tangential vectors $\delta(t, \mathbf{j})$ cannot stay within one and the same subinterval, i.e., at least one subinterval switch must occur over the period $T$. Given the three subintervals $\left[I_{1}\right),\left[I_{2}\right]$, and $\left(I_{3}\right]$, the set of all possible subinterval switches is: (i) switch from $\left[I_{1}\right)$ to $\left[I_{2}\right]$, (ii) switch from $\left[I_{1}\right)$ to $\left(I_{3}\right]$, (iii) switch from $\left[I_{2}\right]$ to $\left[I_{1}\right)$, (iv) switch from $\left[I_{2}\right]$ to $\left(I_{3}\right]$, (v) switch from $\left(I_{3}\right]$ to $\left[I_{1}\right)$, and (vi) switch from $\left(I_{3}\right]$ to $\left[I_{2}\right]$. We analyze now these interval switches. In case (i), i.e., if

(1.) initially, the tangential vector angles are within the interval $\left[I_{1}\right)$ and

(2.) at some later time point, the tangential vector angles switch to the interval $\left[I_{2}\right]$,

$x_{1}(t, \mathbf{j})$ is monotonous (cf. Property 1). This contradicts (9). Analogously, it can be shown that cases (iii), (iv), and (vi) contradict (9), since: in case (iii), $x_{1}(t, \mathbf{j})$ is monotonous; in case (iv), $x_{2}(t, \mathbf{j})$ is monotonous; in case (vi), $x_{2}(t, \mathbf{j})$ is monotonous. Only, in cases (ii) and (v), both, $x_{2}(t, \mathbf{j})$ and $x_{1}(t, \mathbf{j})$, are non-monotonous, which is consistent with (9). In each of the cases (ii) and (v), (12) is true.

(12) $\exists(r, s) \in T^{2} \delta(r, \mathbf{j}) \in\left[I_{1}\right) \wedge \delta(s, \mathbf{j}) \in\left(I_{3}\right]$

The fact that $x_{3}(t, \mathbf{j})$ increases monotonously in sub-scenario (Ca) implies that $\forall t \geq 0 x_{3}(t, \mathbf{j}) \geq x_{3}(0, \mathbf{j})$, where $x_{3}(0, \mathbf{j})=x_{03}$ according to the assumptions made in Proposition 1. In other words, in sub-scenario (Ca), $X\left(T^{+}, \mathbf{j}\right) \subset\left\{\left(x_{1}, x_{2}, x_{3}\right) \in S: x_{3} \geq x_{03}\right\}=: S_{\mathrm{Ca}}\left(\mathbf{x}_{0}\right)$ (cf. Proposition 1). If $X\left(T^{+}, \mathbf{j}\right) \subset S_{\mathrm{Ca}}\left(\mathbf{x}_{0}\right) \Rightarrow X\left(T^{+}, \mathbf{j}\right) \subset S_{1 \sim 3}\left(\mathbf{x}_{0}\right)$, then (13) is valid in sub-scenario $(\mathrm{Ca})$.

(13) $X\left(T^{+}, \mathbf{j}\right) \subset S_{1 \sim 3}\left(\mathbf{x}_{0}\right)$

We prove now that $X\left(T^{+}, \mathbf{j}\right) \subset S_{\mathrm{Ca}}\left(\mathbf{x}_{0}\right) \Rightarrow X\left(T^{+}, \mathbf{j}\right) \subset$ $S_{1 \sim 3}\left(\mathbf{x}_{0}\right)$. Given the point $\mathbf{x}_{0} \equiv\left(x_{01}, x_{02}, x_{03}\right) \in S$ (cf. Proposition 1), the definition of $S_{\mathrm{Ca}}\left(\mathbf{x}_{0}\right)$ (and (1)) implies that (14)-(16) are true if $\mathbf{x}(t, \mathbf{j}) \in S_{\mathrm{Ca}}\left(\mathbf{x}_{0}\right)$.

(14) Either $x_{3}(t, \mathbf{j})>x_{03}$ or $x_{3}(t, \mathbf{j})=x_{03}$ but not both.

(15) $x_{2}(t, \mathbf{j})<x_{02}$ or $x_{2}(t, \mathbf{j})>x_{02}\left(\right.$ or $\left.x_{2}(t, \mathbf{j})=x_{02}\right)$.

(16) $x_{1}(t, \mathbf{j})<x_{01}$ or $x_{1}(t, \mathbf{j})>x_{01}\left(\right.$ or $\left.x_{1}(t, \mathbf{j})=x_{01}\right)$.

The statement (16) can be divided into the two (disjunctive) cases (17a) and (17b).

(17a) Either $x_{1}(t, \mathbf{j})>x_{01}$ or $x_{1}(t, \mathbf{j})=x_{01}$ but not both. (17b) $x_{1}(t, \mathbf{j})<x_{01}$

If (14), (15), and (17a) are true and $\mathbf{x}(t, \mathbf{j}) \in S$, then $\mathbf{x}(t, \mathbf{j}) \in\left\{\left(x_{1}, x_{2}, x_{3}\right) \in S: x_{1} \geq x_{01} \wedge x_{3} \geq x_{03}\right\}$ and, thus, 
$\mathbf{x}(t, \mathbf{j}) \in S_{3}\left(\mathbf{x}_{0}\right)$ (cf. (7f)). We consider now the cases in which (14), (15), and (17b) are true. These cases are:
(18a) $x_{1}(t, \mathbf{j})<x_{01} \wedge x_{3}(t, \mathbf{j})>x_{03} \wedge x_{2}(t, \mathbf{j})>x_{02}$
(18b) $x_{1}(t, \mathbf{j})<x_{01} \wedge x_{3}(t, \mathbf{j})>x_{03} \wedge x_{2}(t, \mathbf{j})<x_{02}$
(18c) $x_{1}(t, \mathbf{j})<x_{01} \wedge x_{3}(t, \mathbf{j})>x_{03} \wedge x_{2}(t, \mathbf{j})=x_{02}$
$(18 \mathrm{~d}) x_{1}(t, \mathbf{j})<x_{01} \wedge x_{3}(t, \mathbf{j})=x_{03} \wedge x_{2}(t, \mathbf{j})>x_{02}$
$(18 \mathrm{e}) x_{1}(t, \mathbf{j})<x_{01} \wedge x_{3}(t, \mathbf{j})=x_{03} \wedge x_{2}(t, \mathbf{j})<x_{02}$
(18f) $x_{1}(t, \mathbf{j})<x_{01} \wedge x_{3}(t, \mathbf{j})=x_{03} \wedge x_{2}(t, \mathbf{j})=x_{02}$

Obviously, the cases (18e) and (18f) violate (1). Thus, if (18e) or (18f) is true, then $\mathbf{x}(t, \mathbf{j}) \notin S$. If (18b) or $(18 \mathrm{c})$ is true and $\mathbf{x}(t, \mathbf{j}) \in S$, then $\mathbf{x}(t, \mathbf{j}) \in\left\{\left(x_{1}, x_{2}, x_{3}\right) \in\right.$ $\left.S: x_{1}<x_{01} \wedge x_{2} \leq x_{02} \wedge x_{3}>x_{03}\right\}=: S_{\mathrm{bc}}\left(\mathbf{x}_{0}\right)$. If $\mathbf{x}(t, \mathbf{j}) \in$ $S_{2}\left(\mathbf{x}_{0}\right)$, then $x_{3}(t, \mathbf{j}) \geq x_{03}$, since, otherwise, (1) is violated (cf. (7d)). In other words, $S_{2}\left(\mathbf{x}_{0}\right)=\left\{\left(x_{1}, x_{2}, x_{3}\right)\right.$ $\left.\in S: x_{1} \leq x_{01} \wedge x_{2} \leq x_{02} \wedge x_{3} \geq x_{03}\right\}$. Obviously, $S_{\mathrm{bc}}\left(\mathbf{x}_{0}\right) \subset$ $S_{2}\left(\mathbf{x}_{0}\right)$. Thus, if (18b) or (18c) is true and $\mathbf{x}(t, \mathbf{j}) \in S$, then $\mathbf{x}(t, \mathbf{j}) \in S_{2}\left(\mathbf{x}_{0}\right)$. Analogously, if (18a), (18c), or (18d) is true and $\mathbf{x}(t, \mathbf{j}) \in S$, then $\mathbf{x}(t, \mathbf{j}) \in\left\{\left(x_{1}, x_{2}, x_{3}\right) \in\right.$ $\left.S: x_{1}<x_{01} \wedge x_{2} \geq x_{02} \wedge x_{3} \geq x_{03}\right\}=: S_{\text {acd }}\left(\mathbf{x}_{0}\right)$. Moreover, if $\mathbf{x}(t, \mathbf{j}) \in S_{1}\left(\mathbf{x}_{0}\right)$, then $x_{1}(t, \mathbf{j}) \leq x_{01}$, since, otherwise, (1) is violated (cf. (7b)). In other words, $S_{1}\left(\mathbf{x}_{0}\right)=\left\{\left(x_{1}, x_{2}, x_{3}\right)\right.$ $\left.\in S: x_{1} \leq x_{01} \wedge x_{2} \geq x_{02} \wedge x_{3} \geq x_{03}\right\}$. Obviously, $S_{\text {acd }}\left(\mathbf{x}_{0}\right) \subset$ $S_{1}\left(\mathbf{x}_{0}\right)$. Thus, if (18a), (18c), or (18d) is true and $\mathbf{x}(t, \mathbf{j}) \in$ $S$, then $\mathbf{x}(t, \mathbf{j}) \in S_{1}\left(\mathbf{x}_{0}\right)$. Overall, we have shown that if $\mathbf{x}(t, \mathbf{j}) \in S_{\mathrm{Ca}}\left(\mathbf{x}_{0}\right) \subseteq S$, then the statements (14)-(17) are valid, which imply several feasible cases. In each of these cases, $\mathbf{x}(t, \mathbf{j})$ is in one of the sets $S_{1}\left(\mathbf{x}_{0}\right), S_{2}\left(\mathbf{x}_{0}\right)$, and $S_{3}\left(\mathbf{x}_{0}\right)$, i.e., $\mathbf{x}(t, \mathbf{j}) \in S_{\mathrm{Ca}}\left(\mathbf{x}_{0}\right) \Rightarrow \mathbf{x}(t, \mathbf{j}) \in S_{1}\left(\mathbf{x}_{0}\right) \cup S_{2}\left(\mathbf{x}_{0}\right) \cup$ $S_{3}\left(\mathbf{x}_{0}\right)$. This implies that $X\left(T^{+}, \mathbf{j}\right) \subset S_{\mathrm{Ca}}\left(\mathbf{x}_{0}\right) \Rightarrow X\left(T^{+}, \mathbf{j}\right)$ $\subset S_{1}\left(\mathbf{x}_{0}\right) \cup S_{2}\left(\mathbf{x}_{0}\right) \cup S_{3}\left(\mathbf{x}_{0}\right)$, since $X\left(T^{+}, \mathbf{j}\right)$ is the union of the points $\mathbf{x}(t, \mathbf{j}) \in S$ for which the statements (14)-(17) (and (1)) hold (cf. Proposition 1). According to (7n), $S_{1 \sim 3}\left(\mathbf{x}_{0}\right)=S_{1}\left(\mathbf{x}_{0}\right) \cup S_{2}\left(\mathbf{x}_{0}\right) \cup S_{3}\left(\mathbf{x}_{0}\right)$. This completes the proof that $X\left(T^{+}, \mathbf{j}\right) \subset S_{\mathrm{Ca}}\left(\mathbf{x}_{0}\right) \Rightarrow X\left(T^{+}, \mathbf{j}\right) \subset S_{1 \sim 3}\left(\mathbf{x}_{0}\right)$.

By now, we have shown that in the sub-scenario (Ca), the statements (8), (12), and (13) must be true. These three statements reduce to Condition Set $\mathrm{P}_{2}$. It can be shown in the same way that

(1.) the sub-scenarios $(\mathrm{Cb})$ and $(\mathrm{Cc})$ correspond to Condition Sets $\mathrm{P} 1_{5}$ and $\mathrm{P} 1_{7}$, respectively, and
(2.) each of the sub-scenarios $(\mathrm{Ba})-(\mathrm{Cc})$ corresponds to one and only one of the Condition Sets $\mathrm{P} 1_{1}, \mathrm{P} 1_{3}, \mathrm{P} 1_{4}$, $\mathrm{P} 1_{6}, \mathrm{P} 1_{8}$, and $\mathrm{P} 1_{9}$.

This completes the proof that each of the alternative (sub-)scenarios of monotonicity in one dimension (i.e., each of the sub-scenarios (Aa)-(Cc)) corresponds to one and only one of the Condition Sets $\mathrm{P} 1_{1}-\mathrm{P} 1_{9}$.

Proposition 2. Assume that (a) the trajectory $X(T, \mathbf{j})$ defined by (4a) is continuous and monotonous in two dimensions on $S$, (b) $\mathbf{x}(0, \mathbf{j})=\mathbf{x}_{0} \equiv\left(x_{01}, x_{02}, x_{03}\right) \in S$, and (c) $\forall t \in T \dot{\mathbf{x}}(t, \mathbf{j}) \neq 0$. Then, $X(T, \mathbf{j})$ and $X\left(T^{+}, \mathbf{j}\right)$ (cf. (4b)) satisfy one and only one of the Condition Sets $\mathrm{P} 2_{1}-\mathrm{P} 2_{6}$, where for $i \in\{1,2, \ldots, 6\}$, Condition Set $\mathrm{P} 2_{i}$ is: $\left(\forall t \in T \delta(t, \mathbf{j}) \in\left[I_{i \sim(i+1)}\right]\right) \wedge\left(\exists(r, s) \in T^{2} \delta(r, \mathbf{j}) \in\left[I_{i}\right)\right.$ $\left.\wedge \delta(s, \mathbf{j}) \in\left(I_{(i+1)}\right]\right) \wedge X\left(T^{+}, \mathbf{j}\right) \subset S_{i \sim(i+1)}\left(\mathbf{x}_{0}\right)(\mathrm{cf} .(6) /(7))$.

Proof. According to the definition of monotonicity in two dimensions, two of the functions $x_{1}(t, \mathbf{j}), x_{2}(t, \mathbf{j})$, and $x_{3}(t, \mathbf{j})$ must be monotonous, while the remaining one must be non-monotonous. Thus, we have to consider only three cases: (A) $x_{1}(t, \mathbf{j})$ and $x_{2}(t, \mathbf{j})$ are monotonous (while $x_{3}(t, \mathbf{j})$ is non-monotonous), (B) $x_{1}(t, \mathbf{j})$ and $x_{3}(t, \mathbf{j})$ are monotonous (while $x_{2}(t, \mathbf{j})$ is non-monotonous), and (C) $x_{2}(t, \mathbf{j})$ and $x_{3}(t, \mathbf{j})$ are monotonous (while $x_{1}(t, \mathbf{j})$ is non-monotonous). For each of these cases, we must distinguish between four subcases. For example, in case (A), we can distinguish between the following subcases: (a) $x_{1}(t, \mathbf{j})$ and $x_{2}(t, \mathbf{j})$ are monotonously increasing, (b) $x_{1}(t, \mathbf{j})$ is monotonously increasing, while $x_{2}(t, \mathbf{j})$ is monotonously decreasing, (c) $x_{1}(t, \mathbf{j})$ and $x_{2}(t, \mathbf{j})$ are monotonously decreasing, and (d) $x_{1}(t, \mathbf{j})$ is monotonously decreasing, while $x_{2}(t, \mathbf{j})$ is monotonously increasing. Subcases (a) and (c) are infeasible, since they violate (1): for example, if $x_{1}(t, \mathbf{j})$ and $x_{2}(t, \mathbf{j})$ are monotonously increasing, then $x_{3}(t, \mathbf{j})$ must be monotonously decreasing (instead of being non-monotonous), since $x_{1}(t, \mathbf{j})+x_{2}(t$, j) $+x_{3}(t, \mathbf{j})$ must be equal to 1 for all $t$. Thus, we must consider only the subcases (b) and (d) of case (A). 
Properties 1 and 2 imply that in subcase (b) of case (A), the statement (19) is valid (cf. (6)).

(19) $\forall t \in T \delta(t, \mathbf{j}) \in\left[I_{3 \sim 4}\right]$

Moreover, since case (A) requires that $x_{3}(t, \mathbf{j})$ is non-monotonous, Property 3 implies that (20) is valid in case (A).

(20) $\exists(r, s) \in T^{2} \dot{x}_{3}(r, \mathbf{j})>0 \wedge \dot{x}_{3}(s, \mathbf{j})<0$

According to (6), the interval $\left[I_{3 \sim 4}\right]$, to which (19) refers, can be partitioned into the following partitions: $\left[I_{3}\right), \alpha_{4}$, and $\left(I_{4}\right]$. Property 3 and (6) imply (21).

(21a) $\forall t \in T \delta(t, \mathbf{j}) \in\left[I_{3}\right) \vee \delta(t, \mathbf{j}) \in\left[I_{3}\right) \cup \alpha_{4} \Rightarrow \forall t \in T$ $\dot{x}_{3}(t, \mathbf{j}) \geq 0$

(21b) $\forall t \in T \delta(t, \mathbf{j}) \in\left(I_{4}\right] \vee \delta(t, \mathbf{j}) \in \alpha_{4} \cup\left(I_{4}\right] \Rightarrow \forall t \in T$ $\dot{x}_{3}(t, \mathbf{j}) \leq 0$

(21c) $\forall t \in T \delta(t, \mathbf{j}) \in \alpha_{4} \Rightarrow \forall t \in T \dot{x}_{3}(t, \mathbf{j})=0$

The statements (21) imply that if (19) and (20) are true, the tangential vectors $\delta(t, \mathbf{j})$ cannot stay within one and only one of the subintervals $\left[I_{3}\right),\left[I_{3}\right) \cup \alpha_{4}, \alpha_{4}, \alpha_{4} \cup$ ( $\left.I_{4}\right]$, and $\left(I_{4}\right]$ for all $t \in T$. That is, there must occur a switch from subinterval $\left[I_{3}\right)$ to subinterval $\left(I_{4}\right]$ or from subinterval $\left(I_{4}\right]$ to subinterval $\left[I_{3}\right)$ over the period $T$. Thus, (22) is valid.

(22) $\exists(r, s) \in T^{2} \delta(r, \mathbf{j}) \in\left[I_{3}\right) \wedge \delta(s, \mathbf{j}) \in\left(I_{4}\right]$

Since in subcase (b) of case (A), $x_{1}(t, \mathbf{j})$ increases monotonously and $x_{2}(t, \mathbf{j})$ decreases monotonously, the assumptions made in Proposition 2 and (4b) imply that $X\left(T^{+}, \mathbf{j}\right) \subset\left\{\left(x_{1}, x_{2}, x_{3}\right) \in S: x_{1} \geq x_{01} \wedge x_{2} \leq x_{02}\right\}=:$ $S_{\mathrm{Ab}}\left(\mathbf{x}_{0}\right) . S_{\mathrm{Ab}}\left(\mathbf{x}_{0}\right)$ can be partitioned as follows: $S_{\mathrm{Ab}}\left(\mathbf{x}_{0}\right)=$ $S_{\mathrm{Ab} 1}\left(\mathbf{x}_{0}\right) \cup S_{\mathrm{Ab} 2}\left(\mathbf{x}_{0}\right)$, where $S_{\mathrm{Ab} 1}\left(\mathbf{x}_{0}\right) \cap S_{\mathrm{Ab} 2}\left(\mathbf{x}_{0}\right)=\emptyset$ and $S_{\mathrm{Ab} 1}\left(\mathbf{x}_{0}\right):=\left\{\left(x_{1}, x_{2}, x_{3}\right) \in S: x_{1} \geq x_{01} \wedge x_{2} \leq x_{02} \wedge x_{3} \geq x_{03}\right\}$ and $S_{\mathrm{Ab} 2}\left(\mathbf{x}_{0}\right):=\left\{\left(x_{1}, x_{2}, x_{3}\right) \in S: x_{1} \geq x_{01} \wedge x_{2} \leq x_{02} \wedge x_{3}<\right.$ $\left.x_{03}\right\}$. We can see immediately that $S_{\mathrm{Ab} 1}\left(\mathbf{x}_{0}\right) \subset S_{3}\left(\mathbf{x}_{0}\right)$ (cf. (7f)) and $S_{\mathrm{Ab} 2}\left(\mathbf{x}_{0}\right) \subset S_{4}\left(\mathbf{x}_{0}\right)$ (cf. (7h)). Thus, $S_{\mathrm{Ab}}\left(\mathbf{x}_{0}\right) \subset$ $S_{3}\left(\mathbf{x}_{0}\right) \cup S_{4}\left(\mathbf{x}_{0}\right)$. This result, (7n), and the previously shown fact that $X\left(T^{+}, \mathbf{j}\right) \subset S_{\mathrm{Ab}}\left(\mathbf{x}_{0}\right)$ imply (23).

(23) $X\left(T^{+}, \mathbf{j}\right) \subset S_{3 \sim 4}\left(\mathbf{x}_{0}\right)$

Overall, we have shown that in the subcase (b) of case (A), Condition Set $\mathrm{P} 2_{3}$ must be true (cf. (19), (22), and (23)). Analogously, it can be shown that in all the feasible subcases of cases (A)-(C), one and only one of the statements $\mathrm{P} 2_{1}, \mathrm{P} 2_{2}, \mathrm{P} 2_{4}, \mathrm{P} 2{ }_{5}$, and $\mathrm{P} 2_{6}$ is true, which proves Proposition 2.

Proposition 3. Assume that (a) the trajectory $X(T, \mathbf{j})$ defined by (4a) is continuous and monotonous (in three dimensions) on $S$, (b) $\mathbf{x}(0, \mathbf{j})=\mathbf{x}_{0} \equiv\left(x_{01}, x_{02}, x_{03}\right) \in S$, and (c) $\forall t \in T \dot{\mathbf{x}}(t, \mathbf{j}) \neq 0$. Then, $X(T, \mathbf{j})$ and $X\left(T^{+}, \mathbf{j}\right)$ (cf. (4b)) satisfy one and only one of the Condition Sets $\mathrm{P} 3_{1}-\mathrm{P} 3_{12}$, where (cf. (6) and (7)):

a) for $i \in\{1,2, \ldots, 6\}$, Condition Set $\mathrm{P} 3_{i}$ is: $(\forall t \in T$ $\left.\delta(t, \mathbf{j}) \in\left[I_{i}\right]\right) \wedge\left(\exists s \in T \delta(s, \mathbf{j}) \in I_{i}\right) \wedge X\left(T^{+}, \mathbf{j}\right) \subset S_{i}\left(\mathbf{x}_{0}\right) ;$

b) for $i \in\{7,8, \ldots, 12\}$, Condition Set $\mathrm{P}_{i}$ is: $\forall t \in T$ $\delta(t, \mathbf{j}) \in\left\{\alpha_{i-6}\right\} \wedge X\left(T^{+}, \mathbf{j}\right) \subseteq L_{i-6}\left(\mathbf{x}_{0}\right)$.

Proof. According to our definition of monotonicity (in three dimensions), $x_{1}(t, \mathbf{j}), x_{2}(t, \mathbf{j})$, and $x_{3}(t, \mathbf{j})$ must be monotonous if $X(T, \mathbf{j})$ is monotonous (in three dimensions) on $S$. Since a monotonous function can be (a) monotonously increasing, (b) monotonously decreasing, or (c) both (monotonously increasing and monotonously decreasing and, thus, constant), we have per function $x_{i}(t, \mathbf{j})$ three cases ((a)-(c)). Moreover, we have three functions $x_{1}(t, \mathbf{j}), x_{2}(t, \mathbf{j})$, and $x_{3}(t, \mathbf{j})$. Thus, overall, there are $3^{3}$ possible combinations. This set of 27 combinations contains the combination (A) $\forall i \dot{x}_{i}(t$, $\mathbf{j}) \leq 0$, the combination (B) $\forall i \dot{x}_{i}(t, \mathbf{j}) \geq 0$, three times the combination (C) $\dot{x}_{i}(t, \mathbf{j}) \geq 0 \wedge \dot{x}_{k}(t, \mathbf{j}) \geq 0 \wedge \dot{x}_{l}(t, \mathbf{j})=0 \wedge i$ $\neq k \neq l$, three times the combination (D) $\dot{x}_{i}(t, \mathbf{j}) \leq 0 \wedge$ $\dot{x}_{k}(t, \mathbf{j}) \leq 0 \wedge \dot{x}_{l}(t, \mathbf{j})=0 \wedge i \neq k \neq l$, six times the combination $(\mathrm{E}) \dot{x}_{i}(t, \mathbf{j})=\dot{x}_{k}(t, \mathbf{j})=0 \wedge \dot{x}_{l}(t, \mathbf{j}) \neq 0 \wedge i \neq k$ $\neq l$, and the combination (F) $\forall i \dot{x}_{i}(t, \mathbf{j})=0$. The combinations (A)-(E) are infeasible, since they violate (1) unless they reduce to combination $(F)$. The combination (F) represents a fixed point $(\dot{\mathbf{x}}(t, \mathbf{j})=0)$ and is excluded by the assumptions made in Proposition 3. In the rest of the proof, we have to consider the remaining 12 combinations. ${ }^{1}$ Each of these 12 combi-

\footnotetext{
1 These feasible combinations are: (1.) $\dot{x}_{1} \leq 0 \wedge \dot{x}_{2} \leq 0 \wedge \dot{x}_{3} \geq 0$, (2.) $\dot{x}_{1} \leq 0 \wedge \dot{x}_{2} \geq 0 \wedge \dot{x}_{3} \leq 0$, (3.) $\dot{x}_{1} \leq 0 \wedge \dot{x}_{2} \geq 0 \wedge \dot{x}_{3} \geq 0$, (4.) $\dot{x}_{1}$ $\leq 0 \wedge \dot{x}_{2} \geq 0 \wedge \dot{x}_{3}=0$, (5.) $\dot{x}_{1} \leq 0 \wedge \dot{x}_{2}=0 \wedge \dot{x}_{3} \geq 0$, (6.) $\dot{x}_{1} \geq 0 \wedge$ $\dot{x}_{2} \leq 0 \wedge \dot{x}_{3} \leq 0$, (7.) $\dot{x}_{1} \geq 0 \wedge \dot{x}_{2} \leq 0 \wedge \dot{x}_{3} \geq 0$, (8.) $\dot{x}_{1} \geq 0 \wedge \dot{x}_{2} \leq 0$ $\wedge \dot{x}_{3}=0$, (9.) $\dot{x}_{1} \geq 0 \wedge \dot{x}_{2} \geq 0 \wedge \dot{x}_{3} \leq 0$, (10.) $\dot{x}_{1} \geq 0 \wedge \dot{x}_{2}=0 \wedge \dot{x}_{3} \leq$ 0 , (11.) $\dot{x}_{1}=0 \wedge \dot{x}_{2} \leq 0 \wedge \dot{x}_{3} \geq 0$, and (12.) $\dot{x}_{1}=0 \wedge \dot{x}_{2} \geq 0 \wedge \dot{x}_{3} \leq$
} 
nations is covered by one of the Conditions Sets $\mathrm{P} 3_{1^{-}}$ $\mathrm{P} 3_{12}$. We leave it to the reader to prove the validity of Proposition 3 in all these 12 cases; we prove the validity in only two representative cases. Consider the case $\forall t \in T \dot{x}_{1}(t, \mathbf{j}) \leq 0 \wedge \dot{x}_{2}(t, \mathbf{j}) \leq 0 \wedge \dot{x}_{3}(t, \mathbf{j}) \geq 0$, where $\exists(r, s, p) \in T^{3} \dot{x}_{1}(r, \mathbf{j})<0 \wedge \dot{x}_{2}(s, \mathbf{j})<0 \wedge \dot{x}_{3}(p, \mathbf{j})>0$. Then,

(a) Properties 1-3 imply almost directly that the tangential vector angles $\delta(t, \mathbf{j})$ satisfy the Condition Set $\mathrm{P}_{2}$, and

(b) the assumptions made in Proposition 3 and (4b) imply that $X\left(T^{+}, \mathbf{j}\right) \subset\left\{\left(x_{1}, x_{2}, x_{3}\right) \in S: x_{1} \leq x_{01} \wedge x_{2} \leq x_{02}\right.$ $\left.\wedge x_{3} \geq x_{03}\right\}=: S_{\mathrm{z}}\left(\mathbf{x}_{0}\right)$, and, thus, (7d) implies that $S_{\mathrm{z}}\left(\mathbf{x}_{0}\right)$ $\subset S_{2}\left(\mathbf{x}_{0}\right)$; thus, $X\left(T^{+}, \mathbf{j}\right) \subset S_{2}\left(\mathbf{x}_{0}\right)$ as stated by the Condition Set $\mathrm{P} 3_{2}$.

Alternatively, consider the case $\forall t \in T \dot{x}_{1}(t, \mathbf{j}) \leq 0 \wedge$ $\dot{x}_{2}(t, \mathbf{j}) \geq 0 \wedge \dot{x}_{3}(t, \mathbf{j})=0$, where $\exists r \in T \dot{x}_{1}(r, \mathbf{j})<0 \wedge$ $\dot{x}_{2}(r, \mathbf{j})>0 .^{2}$ Properties 1-3 imply almost directly that in this case, the tangential vector angles $\delta(t, \mathbf{j})$ satisfy the Condition Set $\mathrm{P} 3_{7}$. Moreover, the assumptions made in Proposition 3, (4b), and (7a) imply that $X\left(T^{+}\right.$, j) $\subseteq\left\{\left(x_{1}, x_{2}, x_{3}\right) \in S: x_{1} \leq x_{01} \wedge x_{2} \geq x_{02} \wedge x_{3}=x_{03}\right\} \subseteq$ $L_{1}\left(\mathbf{x}_{0}\right)$. Thus, $X\left(T^{+}, \mathbf{j}\right) \subseteq L_{1}\left(\mathbf{x}_{0}\right)$ as stated by Condition Set $\mathrm{P} 3_{7}$.

We discuss now the geometrical interpretation of Properties 1-3 as depicted by Figure 4. To construct Figure 4 , we choose an arbitrary point $\left(\mathbf{x}_{0}\right)$ in the interior of $S$. Then, we draw three line segments going through $\mathbf{x}_{0}$ and each being parallel to one of the simplex edges $\mathbf{v}_{1}-\mathbf{v}_{2}, \mathbf{v}_{2}-\mathbf{v}_{3}$, and $\mathbf{v}_{3}-\mathbf{v}_{1}$. The intersections of the line segments with the simplex edges are denoted by the points a-f. We can see that the line segments that connect $\mathbf{x}_{0}$ with one of the points a-f are the line segments $L_{1}\left(\mathbf{x}_{0}\right)-L_{6}\left(\mathbf{x}_{0}\right)$, which are defined by (7) and which localize the six (closed) subsets $S_{1}\left(\mathbf{x}_{0}\right)-S_{6}\left(\mathbf{x}_{0}\right)$ defined by (7). The angles between the line segments

0 , where $\exists t \in T \dot{x}_{i}(t)<0$ if it is stated that $\dot{x}_{i} \leq 0$, and, analogously, $\exists t \in T \dot{x}_{i}(t)>0$ if it is stated that $\dot{x}_{i} \geq 0$.

${ }^{2}$ Note that the cases $\dot{x}_{1}(r, \mathbf{j})<0 \wedge \dot{x}_{2}(r, \mathbf{j})=\dot{x}_{3}(r, \mathbf{j})=0$ and $\dot{x}_{2}(r$, $\mathbf{j})>0 \wedge \dot{x}_{1}(r, \mathbf{j})=\dot{x}_{3}(r, \mathbf{j})=0$ are infeasible (see the discussion of combinations (A)-(E)).
$L_{1}\left(\mathbf{x}_{0}\right)-L_{6}\left(\mathbf{x}_{0}\right)$ and the simplex edge $\mathbf{v}_{1}-\mathbf{v}_{2}$ (according to the definition of tangential vector angles and intervals (6)) are depicted in the middle panel of Figure 4. (7n) and Figure 4 imply almost directly that

(1.) each of the six sets $S_{i \sim(i+1)}\left(\mathbf{x}_{0}\right)$, to which Proposition 2 refers, is simply the union of two neighboring sets $S_{j}\left(\mathbf{x}_{0}\right)$ and $S_{k}\left(\mathbf{x}_{0}\right)$,

(2.) each of the six sets $S_{(i-1) \sim(i+1)}\left(\mathbf{x}_{0}\right)$, to which Proposition 1 refers, is simply the union of three neighboring sets $S_{j}\left(\mathbf{x}_{0}\right), S_{k}\left(\mathbf{x}_{0}\right)$, and $S_{m}\left(\mathbf{x}_{0}\right)$.

In particular, Propositions 1-3 can be interpreted easily by using Figure 4:

1.) Proposition 3 implies three geometrical properties of a trajectory segment $X\left(T^{+}, \mathbf{j}\right)$ that is monotonous in three dimensions. First, $X\left(T^{+}, \mathbf{j}\right)$ is located in one of the line segments $L_{1}\left(\mathbf{x}_{0}\right)-L_{6}\left(\mathbf{x}_{0}\right)$ or in one of the sets $S_{1}\left(\mathbf{x}_{0}\right)-S_{6}\left(\mathbf{x}_{0}\right)$. Second, if $X\left(T^{+}, \mathbf{j}\right)$ is in $L_{i}\left(\mathbf{x}_{0}\right)$, then for all $t \geq 0$, the tangential vector angles $\delta(t, \mathbf{j})$ associated with $X\left(T^{+}, \mathbf{j}\right)$ are equal to the angle that is associated to the line segment $L_{i}\left(\mathbf{x}_{0}\right)$ in Figure $4+/-180^{\circ}$. For example, if $X\left(T^{+}, \mathbf{j}\right)$ is in $L_{3}\left(\mathbf{x}_{0}\right)$, then $\delta(t, \mathbf{j}) \in\left\{120^{\circ}, 300^{\circ}\right\}$ for $t \geq 0$. Third, if $X\left(T^{+}, \mathbf{j}\right)$ is located in one of the sets $S_{i}\left(\mathbf{x}_{0}\right)$, then for $t \geq 0$, the tangential vector angles $\delta(t, \mathbf{j})$ associated with $X\left(T^{+}, \mathbf{j}\right)$ are within the angle range indicated by the angles associated to the line segments $L_{i}\left(\mathbf{x}_{0}\right)$ and $L_{i+1}\left(\mathbf{x}_{0}\right)$ that bound the set $S_{i}\left(\mathbf{x}_{0}\right)$ in Figure 4. For example, if the trajectory segment $X\left(T^{+}, \mathbf{j}\right)$ that is monotonous in three dimensions is in $S_{3}\left(\mathbf{x}_{0}\right)$, then $\delta(t, \mathbf{j})$ is within the angle range $\left[120^{\circ}, 180^{\circ}\right]$ for $t \geq 0$ (cf. Proposition 3 and Condition Set $\mathrm{P} 3_{3}$ ).

2.) The geometrical interpretation of Proposition 2 is analogous. In particular, the trajectory segment $X\left(T^{+}, \mathbf{j}\right)$ that is monotonous in two dimensions is located in two neighboring sets $S_{j}\left(\mathbf{x}_{0}\right)$ and $S_{k}\left(\mathbf{x}_{0}\right)$, and for all $t \geq 0$, the tangential vector angles $\delta(t, \mathbf{j})$ of $X\left(T^{+}, \mathbf{j}\right)$ are within the angle range indicated by the angles associated to the two line segments $L_{j}\left(\mathbf{x}_{0}\right)$ and $L_{k+1}\left(\mathbf{x}_{0}\right)$ that bound the union of the sets $S_{j}\left(\mathbf{x}_{0}\right)$ and $S_{k}\left(\mathbf{x}_{0}\right)$ in Figure 4. For example, if the trajectory segment $X\left(T^{+}, \mathbf{j}\right)$ that is monotonous in two dimensions is in $S_{3 \sim 4}\left(\mathbf{x}_{0}\right)=S_{3}\left(\mathbf{x}_{0}\right) \cup$ 
$S_{4}\left(\mathbf{x}_{0}\right)$, then $\delta(t, \mathbf{j})$ is within the angle range $\left[120^{\circ}, 240^{\circ}\right]$ for $t \geq 0$ (cf. Proposition 2 and Condition Set $\mathrm{P} 2_{3}$ ).

3.) Analogously, Proposition 1 implies that the trajectory segment $X\left(T^{+}, \mathbf{j}\right)$ that is monotonous in one dimension is located in three neighboring sets $S_{j}\left(\mathbf{x}_{0}\right)$, $S_{k}\left(\mathbf{x}_{0}\right)$, and $S_{m}\left(\mathbf{x}_{0}\right)$. Moreover, for all $t \geq 0$, the tangential vector angles $\delta(t, \mathbf{j})$ associated with this trajectory segment are within the angle range indicated by the angles associated to the two line segments $L_{j}\left(\mathbf{x}_{0}\right)$ and $L_{m+1}\left(\mathbf{x}_{0}\right)$ that bound the union of the sets $S_{j}\left(\mathbf{x}_{0}\right), S_{k}\left(\mathbf{x}_{0}\right)$, and $S_{m}\left(\mathbf{x}_{0}\right)$ in Figure 4. For example, if the trajectory segment $X\left(T^{+}, \mathbf{j}\right)$ that is monotonous in one dimensions is in $S_{3 \sim 5}\left(\mathbf{x}_{0}\right)=S_{3}\left(\mathbf{x}_{0}\right) \cup S_{4}\left(\mathbf{x}_{0}\right) \cup S_{4}\left(\mathbf{x}_{0}\right)$, then $\delta(t, \mathbf{j})$ is within the angle range $\left[120^{\circ}, 300^{\circ}\right]$ for $t \geq 0$ (cf. Proposition 1 and Condition Set $\mathrm{P}_{4}$ ).

This graphical interpretation highlights important implications of Propositions 1-3: First, a trajectory that is monotonous (in three dimensions) is captured in a smaller subset of $S$ than a related trajectory that is monotonous in two dimensions. Second, a trajectory that is monotonous in two dimensions is captured in a smaller subset of $S$ than a related trajectory that is monotonous in one dimension. Moreover, the maximum curvature $\kappa^{*}$ of trajectories that are monotonous in three dimensions (two dimensions) is greater than the maximum curvature of related trajectories that are monotonous in two dimensions (one dimension).

This intuitive discussion does not explicitly define the meaning of the term 'related'. Thus, we define the meaning of this term and then formulate Corollary 1 (which is implied by Propositions 1-3) on the basis of this definition such that the discussion becomes more precise. Let $F\left(\mathbf{x}_{0}\right) \subseteq J$ be a family of continuous trajectory segments $X\left(T^{+}, \mathbf{j}\right) \subset S, \mathbf{j} \in F\left(\mathbf{x}_{0}\right)$, satisfying $\forall \mathbf{j} \in$ $F\left(\mathbf{x}_{0}\right) \mathbf{x}(0, \mathbf{j})=\mathbf{x}_{0} \in S$ and $\forall t \in T^{+} \forall \mathbf{j} \in F\left(\mathbf{x}_{0}\right) \dot{\mathbf{x}}(t, \mathbf{j}) \neq 0$ (cf. (4)). Moreover, let $P 1_{1}\left(\mathbf{x}_{0}\right), P 1_{2}\left(\mathbf{x}_{0}\right), \ldots, P 1_{6}\left(\mathbf{x}_{0}\right)$, $P 2_{1}\left(\mathbf{x}_{0}\right), P 2_{2}\left(\mathbf{x}_{0}\right), \ldots, P 2_{6}\left(\mathbf{x}_{0}\right), P 3_{1}\left(\mathbf{x}_{0}\right), P 3_{2}\left(\mathbf{x}_{0}\right), \ldots$, and $P 3_{6}\left(\mathbf{x}_{0}\right)$ denote the subfamilies of $F\left(\mathbf{x}_{0}\right)$ satisfying the Conditions Sets $\mathrm{P} 1_{1}, \mathrm{P} 1_{2}, \ldots, \mathrm{P} 1_{6}, \mathrm{P} 2_{1}, \mathrm{P} 2_{2}, \ldots, \mathrm{P} 2_{6}$, $\mathrm{P} 3_{1}, \mathrm{P} 3_{2}, \ldots$, and $\mathrm{P} 3_{6}$, respectively. That is, $\mathbf{j} \in P c_{d}\left(\mathbf{x}_{0}\right)$ $\subset F\left(\mathbf{x}_{0}\right)$ implies that $X\left(T^{+}, \mathbf{j}\right)$ satisfies the Condition Set $\mathrm{P} c_{d}$, where $c \in\{1,2,3\}$ and $d \in\{1,2, \ldots, 6\}$. For $(h, k)$ $\in\{1,2, \ldots, 6\}^{2}$, we say that the families $P 1_{h}\left(\mathbf{x}_{0}\right)$ and $P 2_{k}\left(\mathbf{x}_{0}\right)$ are related if $\exists i \in\{1,2,3\} \forall \mathbf{j} \in P 1_{h}\left(\mathbf{x}_{0}\right) \cup$ $P 2_{k}\left(\mathbf{x}_{0}\right)\left(\forall t \in T^{+} \dot{x}_{i}(t, \mathbf{j}) \geq 0\right) \vee\left(\forall t \in T^{+} \dot{x}_{i}(t, \mathbf{j}) \leq 0\right) \wedge\left(\exists t_{\mathbf{j}}\right.$ $\left.\in T^{+} \dot{x}_{i}\left(t_{\mathbf{j}}, \mathbf{j}\right) \neq 0\right)$. That is, a family defined by Proposition 1 is related to a family defined by Proposition 2 if there exists an $i$ for which the monotonicity characteristics of $x_{i}(t, \mathbf{j})$ are identical in both families. For example, the families $P 1_{2}\left(\mathbf{x}_{0}\right)$ and $P 2_{1}\left(\mathbf{x}_{0}\right)$ are characterized by a monotonously increasing $x_{3}(t, \mathbf{j})$, i.e., $\forall \mathbf{j} \in P 1_{2}\left(\mathbf{x}_{0}\right) \cup P 2_{1}\left(\mathbf{x}_{0}\right)\left(\forall t \in T^{+} \dot{x}_{3}(t, \mathbf{j}) \geq 0\right) \wedge\left(\exists t_{\mathbf{j}} \in\right.$ $\left.T^{+} \dot{x}_{3}\left(t_{\mathbf{j}}, \mathbf{j}\right)>0\right)$; thus, $P 1_{2}\left(\mathbf{x}_{0}\right)$ and $P 2_{1}\left(\mathbf{x}_{0}\right)$ are related. We define the relations between the families defined by Propositions 2 and 3 analogously: For $(p, q) \in\{1,2, \ldots$, $6\}^{2}$, we say that the families $P 2_{p}\left(\mathbf{x}_{0}\right)$ and $P 3_{q}\left(\mathbf{x}_{0}\right)$ are related if $\exists(v, w) \in\{1,2,3\}^{2} \forall \mathbf{j} \in P 2_{p}\left(\mathbf{x}_{0}\right) \cup P 3_{q}\left(\mathbf{x}_{0}\right)$ $\left(\forall t \in T^{+} \dot{x}_{v}(t, \mathbf{j}) \geq 0\right) \vee\left(\forall t \in T^{+} \dot{x}_{v}(t, \mathbf{j}) \leq 0\right) \wedge\left(\forall t \in T^{+}\right.$ $\left.\dot{x}_{w}(t, \mathbf{j}) \geq 0\right) \vee\left(\forall t \in T^{+} \dot{x}_{w}(t, \mathbf{j}) \leq 0\right) \wedge\left(\exists t_{\mathbf{j}} \in T^{+} \dot{x}_{v}\left(t_{\mathbf{j}}, \mathbf{j}\right) \neq\right.$ $0) \wedge\left(\exists s_{\mathbf{j}} \in T^{+} \dot{x}_{i}\left(s_{\mathbf{j}}, \mathbf{j}\right) \neq 0\right) \wedge v \neq w$. That is, a family defined by Proposition 2 is related to a family defined by Proposition 3 if (a) there exists a $v$ for which the monotonicity characteristics of $x_{v}(t, \mathbf{j})$ are identical in both families and (b) there exists a $w \neq v$ for which the monotonicity characteristics of $x_{w}(t, \mathbf{j})$ are identical in both families. For example, as implied by (6), Properties 1 and 3, and Propositions 2 and 3, the families $P 2_{1}\left(\mathbf{x}_{0}\right)$ and $P 3_{1}\left(\mathbf{x}_{0}\right)$ are characterized by (a) a monotonously decreasing $x_{1}(t, \mathbf{j})$, i.e., $\forall \mathbf{j} \in P 2_{1}\left(\mathbf{x}_{0}\right) \cup$ $P 3_{1}\left(\mathbf{x}_{0}\right)\left(\forall t \in T^{+} \dot{x}_{1}(t, \mathbf{j}) \leq 0\right) \wedge\left(\exists t_{\mathbf{j}} \in T^{+} \dot{x}_{1}\left(t_{\mathbf{j}}, \mathbf{j}\right)<0\right)$, and (b) a monotonously increasing $x_{3}(t, \mathbf{j})$, i.e., $\forall \mathbf{j} \in$ $P 2_{1}\left(\mathbf{x}_{0}\right) \cup P 3_{1}\left(\mathbf{x}_{0}\right)\left(\forall t \in T^{+} \dot{x}_{3}(t, \mathbf{j}) \geq 0\right) \wedge\left(\exists s_{\mathbf{j}} \in T^{+} \dot{x}_{3}\left(s_{\mathbf{j}}\right.\right.$, j) > 0). Thus, $P 2_{1}\left(\mathbf{x}_{0}\right)$ and $P 3_{1}\left(\mathbf{x}_{0}\right)$ are related.

Corollary 1. a) Consider the trajectory family $P 1_{h}\left(\mathbf{x}_{0}\right)$, where $h \in\{1,2, \ldots, 6\}$ and $\mathbf{x}_{0} \equiv\left(x_{01}, x_{02}, x_{03}\right) \in$ $\operatorname{int}(S)$. There exist two trajectory families $P 2_{k}\left(\mathbf{x}_{0}\right)$ and $P 2_{m}\left(\mathbf{x}_{0}\right),(k, m) \in\{1,2, \ldots, 6\}^{2}, k \neq m$, that are related to $P 1_{h}\left(\mathbf{x}_{0}\right)$ and satisfy the following condition: $\forall n \in\{k$, m) $a^{*}\left(T^{+}, P 1_{h}\left(\mathbf{x}_{0}\right)\right)>a^{*}\left(T^{+}, P 2_{n}\left(\mathbf{x}_{0}\right)\right) \wedge \kappa^{*}\left(T^{+}\right.$, $\left.P 1_{h}\left(\mathbf{x}_{0}\right)\right)>\kappa^{*}\left(T^{+}, P 2_{n}\left(\mathbf{x}_{0}\right)\right)$ (cf. Sections 2.2. and 2.3). 
b) Consider the trajectory family $P 2_{p}\left(\mathbf{x}_{0}\right)$, where $p \in$ $\{1,2, \ldots, 6\}$ and $\mathbf{x}_{0} \equiv\left(x_{01}, x_{02}, x_{03}\right) \in \operatorname{int}(S)$. There exist two trajectory families $P 3_{q}\left(\mathbf{x}_{0}\right)$ and $P 3_{r}\left(\mathbf{x}_{0}\right),(q, r) \in\{1$, $2, \ldots, 6\}^{2}, q \neq r$, that are related to $P 2_{p}\left(\mathbf{x}_{0}\right)$ and satisfy the following condition: $\forall u \in\{q, r\} a^{*}\left(T^{+}, P 2_{p}\left(\mathbf{x}_{0}\right)\right)>$ $a^{*}\left(T^{+}, P 3_{u}\left(\mathbf{x}_{0}\right)\right) \wedge \kappa^{*}\left(T^{+}, P 2_{p}\left(\mathbf{x}_{0}\right)\right)>\kappa^{*}\left(T^{+}, P 3_{u}\left(\mathbf{x}_{0}\right)\right)$.

Proof. We only sketch here the proof. Starting with Corollary 1a, assume that $h=2$, i.e., consider the family $P 1_{2}\left(\mathbf{x}_{0}\right)$. According to our definition of relatedness, $P 1_{2}\left(\mathbf{x}_{0}\right)$ is related to $P 2_{1}\left(\mathbf{x}_{0}\right)$ and $P 2_{2}\left(\mathbf{x}_{0}\right)$, since (6), Property 3, and Propositions 1 and 2 imply that $P 1_{2}\left(\mathbf{x}_{0}\right), P 2_{1}\left(\mathbf{x}_{0}\right)$, and $P 2_{2}\left(\mathbf{x}_{0}\right)$ are characterized by a monotonously increasing $x_{3}(t, \mathbf{j})$.

According to Proposition 1, (7b), (7d), (7f), (7n), and the definitions of $a$ and $M$ given in Section 2.2, the following statements are true:

(24) $M\left(T^{+}, P 1_{2}\left(\mathbf{x}_{0}\right)\right) \subseteq S_{1 \sim 3}\left(\mathbf{x}_{0}\right)=S_{1}\left(\mathbf{x}_{0}\right) \cup S_{2}\left(\mathbf{x}_{0}\right) \cup$ $S_{3}\left(\mathbf{x}_{0}\right)=\left\{\left(x_{1}, x_{2}, x_{3}\right) \in S: x_{3} \geq x_{03}\right\}$

(25) $M\left(T^{+}, P 2_{1}\left(\mathbf{x}_{0}\right)\right) \subseteq S_{1 \sim 2}\left(\mathbf{x}_{0}\right)=S_{1}\left(\mathbf{x}_{0}\right) \cup S_{2}\left(\mathbf{x}_{0}\right) \subset$ $S_{1 \sim 3}\left(\mathbf{x}_{0}\right)$

(26) $M\left(T^{+}, P 2_{2}\left(\mathbf{x}_{0}\right)\right) \subseteq S_{2 \sim 3}\left(\mathbf{x}_{0}\right)=S_{2}\left(\mathbf{x}_{0}\right) \cup S_{3}\left(\mathbf{x}_{0}\right) \subset$ $S_{1 \sim 3}\left(\mathbf{x}_{0}\right)$

As implied by (24), all the trajectories belonging to the family $P 1_{2}\left(\mathbf{x}_{0}\right)$ are located in $S_{1 \sim 3}\left(\mathbf{x}_{0}\right)$, where the latter is a triangle obtained by constructing a line on $S$ going through $\mathbf{x}_{0}$ and being parallel to the simplex edge $\mathbf{v}_{1}-\mathbf{v}_{2}$ (cf. Property 3a, (6), and Figures 3 and 5). According to Proposition 1 and (6), all the trajectories belonging to the family $P 1_{2}\left(\mathbf{x}_{0}\right)$ satisfy the following vector angle condition:

(27) $\forall \mathbf{j} \in P 1_{2}\left(\mathbf{x}_{0}\right)\left(\forall t \in T^{+} \delta(t, \mathbf{j}) \in\left[0^{\circ}, 180^{\circ}\right]\right) \wedge(\exists(r$, $s) \in T^{+} \times T^{+} \delta(r, \mathbf{j}) \in\left[0^{\circ}, 60^{\circ}\right) \wedge \delta(s, \mathbf{j}) \in\left(120^{\circ}\right.$, $\left.180^{\circ}\right]$ )

If we allow for non-smooth trajectories and, in particular, trajectories that are unions of line segments, it is easy to show geometrically by referring to Figure 5 that such trajectories can be constructed to any point on $S_{1 \sim 3}\left(\mathbf{x}_{0}\right)$ while satisfying the condition (27). ${ }^{3}$ Thus,

\footnotetext{
${ }^{3}$ Exactly speaking, (a) each of the line segments constituting such a trajectory is characterized by an angle to the $\mathbf{v}_{1}-\mathbf{v}_{2}$-edge of $S$ in the range of $\left[0^{\circ}, 180^{\circ}\right]$, (b) each trajectory contains a
}

$M\left(T^{+}, P 1_{2}\left(\mathbf{x}_{0}\right)\right)=S_{1 \sim 3}\left(\mathbf{x}_{0}\right)$ and, thus, $a^{*}\left(T^{+}, P 1_{2}\left(\mathbf{x}_{0}\right)\right)=$ $a\left(S_{1 \sim 3}\left(\mathbf{x}_{0}\right)\right)$ (cf. Section 2.2). Moreover, (25) and (26) imply that $a^{*}\left(T^{+}, P 2_{1}\left(\mathbf{x}_{0}\right)\right) \leq a\left(S_{1 \sim 2}\left(\mathbf{x}_{0}\right)\right)<a\left(S_{1 \sim 3}\left(\mathbf{x}_{0}\right)\right)$ and $\left.a^{*}\left(T^{+}, P 2_{2}\left(\mathbf{x}_{0}\right)\right)\right) \leq a\left(S_{2 \sim 3}\left(\mathbf{x}_{0}\right)\right)<a\left(S_{1 \sim 3}\left(\mathbf{x}_{0}\right)\right)$. Thus, (28) is true.

(28) $a^{*}\left(T^{+}, P 1_{2}\left(\mathbf{x}_{0}\right)\right)>a^{*}\left(T^{+}, P 2_{1}\left(\mathbf{x}_{0}\right)\right) \wedge a^{*}\left(T^{+}, P 1_{2}\left(\mathbf{x}_{0}\right)\right)$ $>a^{*}\left(T^{+}, P 2_{2}\left(\mathbf{x}_{0}\right)\right)$.

If we require that the trajectories belonging to the family $P 1_{2}\left(\mathbf{x}_{0}\right)$ are smooth (i.e., $\forall \mathbf{j} \in P 1_{2}\left(\mathbf{x}_{0}\right) \forall t \in T^{+}$ $\mathbf{x}(t, \mathbf{j})$ is differentiable with respect to $t)$, then it is not possible to construct a trajectory that obeys (27) and goes through the points/vertices $\left(x_{01}, 0, x_{03}\right) \in S_{1 \sim 3}\left(\mathbf{x}_{0}\right)$ and $\left(0, x_{02}, x_{03}\right) \in S_{1 \sim 3}\left(\mathbf{x}_{0}\right)$, which can be easily proven by referring to Figures 4 and 5 . That is, the smooth trajectories belonging to the family $P 1_{2}\left(\mathbf{x}_{0}\right)$ cannot cover two infinitesimally small areas of $S_{1 \sim 3}\left(\mathbf{x}_{0}\right)$. However, even in this case, it is still ensured that $a^{*}\left(T^{+}\right.$, $\left.P 1_{2}\left(\mathbf{x}_{0}\right)\right)>a^{*}\left(T^{+}, P 2_{1}\left(\mathbf{x}_{0}\right)\right)$, since

(a) $S_{1 \sim 3}\left(\mathbf{x}_{0}\right)=S_{1 \sim 2}\left(\mathbf{x}_{0}\right) \cup S_{3}\left(\mathbf{x}_{0}\right)$ (cf. (24) and (25)),

(b) $S_{3}\left(\mathbf{x}_{0}\right)$ (cf. (24)) is not infinitesimally small (in generic cases), and

(c) $a^{*}\left(T^{+}, P 2_{1}\left(\mathbf{x}_{0}\right)\right) \leq a\left(S_{1 \sim 2}\left(\mathbf{x}_{0}\right)\right)$.

The definition of $\kappa^{*}$ (cf. Section 2.3) and (27) imply that $\kappa^{*}\left(T^{+}, P 1_{2}\left(\mathbf{x}_{0}\right)\right)=180^{\circ}$. Analogously, Proposition 2, definition of $\kappa^{*}$, and (6) imply that $\kappa^{*}\left(T^{+}, P 2_{1}\left(\mathbf{x}_{0}\right)\right)=$ $120^{\circ}$. Thus, $\kappa^{*}\left(T^{+}, P 1_{2}\left(\mathbf{x}_{0}\right)\right)>\kappa^{*}\left(T^{+}, P 2_{1}\left(\mathbf{x}_{0}\right)\right)$.

Overall, by now we have (heuristically) proven Corollary 1a for $h=2$. The proof is analogous for $h \in$ $\{1,3,4,5,6\}$. The proof of Corollary $1 \mathrm{~b}$ is very similar to the proof of Corollary 1a. Thus, we omit it here.

Overall, among related trajectories and trajectory families the following is true: the higher the dimension of monotonicity, the smaller is (a) the family image (and, thus, the set of predicted states) and (b) the maximal curvature and (and, thus, the potential strength of waves).

line segment that has an angle in the range of $\left[0^{\circ}, 60^{\circ}\right.$ ), and (c) each trajectory contains a line segment that has an angle in the range $\left(120^{\circ}, 180^{\circ}\right.$ ] (cf. (27)). 
If two families are unrelated, then a higher degree of monotonicity does not necessarily imply a smaller family image and a smaller maximum curvature.

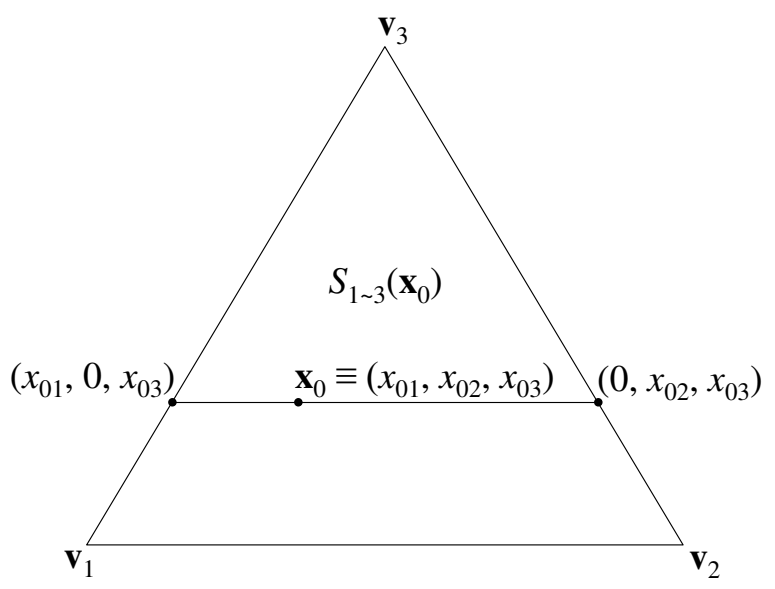

Figure 5. The set $S_{1 \sim 3}\left(\mathbf{x}_{0}\right)$.

We can see that Corollary 1 does not categorize all the Condition Sets postulated by Propositions 1 and 3 and, in particular, not the Condition Sets $\mathrm{P} 1_{7}-\mathrm{P}_{9}$ and $\mathrm{P} 3_{7}-\mathrm{P} 3_{12}$. These Condition Sets imply that one of the $x_{i}(t, \mathbf{j})$ is constant for all $t \in T^{+}$and, thus, the trajectory segments $X\left(T^{+}, \mathbf{j}\right)$ are located on line segments. Obviously, the constancy requirement is much stronger than a monotonicity requirement, thus, in the cases representted by the Condition Sets $\mathrm{P} 1_{7}-\mathrm{P}_{9}$ and $\mathrm{P} 3_{7}-\mathrm{P} 3_{12}$, the size of the family image $M($.$) is relatively small and the$ curvature is zero.

\subsection{Implications for Prediction of Transitional Dynamics}

If we regard $t=0$ as now and $t>0$ as the future (and, thus, $T^{+}$as the predicted trajectory segment), Corollary 1 implies that the trajectories that are monotonous in one dimension (two dimensions) are harder to predict than the trajectories that are monotonous in two dimensions (three dimensions), ceteris paribus, since (a) the set of all possible future states is relatively great and (b) relatively stronger curvatures/waves may arise in the former case (in comparison to the latter case).
However, besides the monotonicity characteristics, the location of the initial state $\mathbf{x}_{0}$ is decisive for the predictability of the future dynamics. In particular, the family image $M$ and its size $a^{*}$ depend on $\mathbf{x}_{0}$ (cf. Corollary 1 and its proof). In general, monotonicity implies that the system moves from $\mathbf{x}_{0}$ along the trajectory segment $T^{+}$towards a vertex or an edge of the 2-simplex. Thus, if the initial state $\mathbf{x}_{0}$ is relatively close to this vertex/edge, $T^{+}$is captured in a relatively small set, i.e., the set of potential future states of the system is relatively small. This is almost a direct implication of the boundedness of the 2-simplex.

As implied by Propositions 1-3, (6), and Section 2.3 (cf. Proof of Corollary 1), the maximum curvature $\kappa^{*}$ of trajectories that are monotonous in three dimensions, two dimensions, and one dimension is $60^{\circ}, 120^{\circ}$, and $180^{\circ}$, respectively. Thus, the cyclical behavior corresponding to a (transversally) self-intersecting or closed trajectory (Jordan curve) is prohibited in all cases of monotonicity, since this type of cyclical behavior requires a curvature greater than $180^{\circ}$. Yet, monotonous trajectories allow for cyclical behavior corresponding to waves on the simplex (see Figure 6).

The angle range of $180^{\circ}$ associated with monotonicity in one dimension allows for waves of high amplitude and short wavelength on the 2-simplex. In contrast, monotonicity in three dimensions allows only for relatively low-amplitude/long-wavelength waves (cf. Figure 6).

Obviously, all three types of monotonicity allow for curved trajectories on the 2-simplex. However, only monotonicity in three dimensions allows for unidirectional linear trajectories, while monotonicity in one dimension allows for linear trajectories yet requires at least one direction change.

\subsection{Limit Dynamics}

In all three cases of monotonicity (monotonicity in one, two, and three dimensions), the following two facts are true for continuous dynamics. First, the cyclical limit dynamics where the omega limit set is a 
Jordan curve are excluded, since such cycles require that $x_{1}(t), x_{2}(t)$, and $x_{3}(t)$ are non-monotonous in the limit. Second, the system may converge to a fixed point or reach the fixed point in finite time (and stay there). The proof of these facts is obvious.
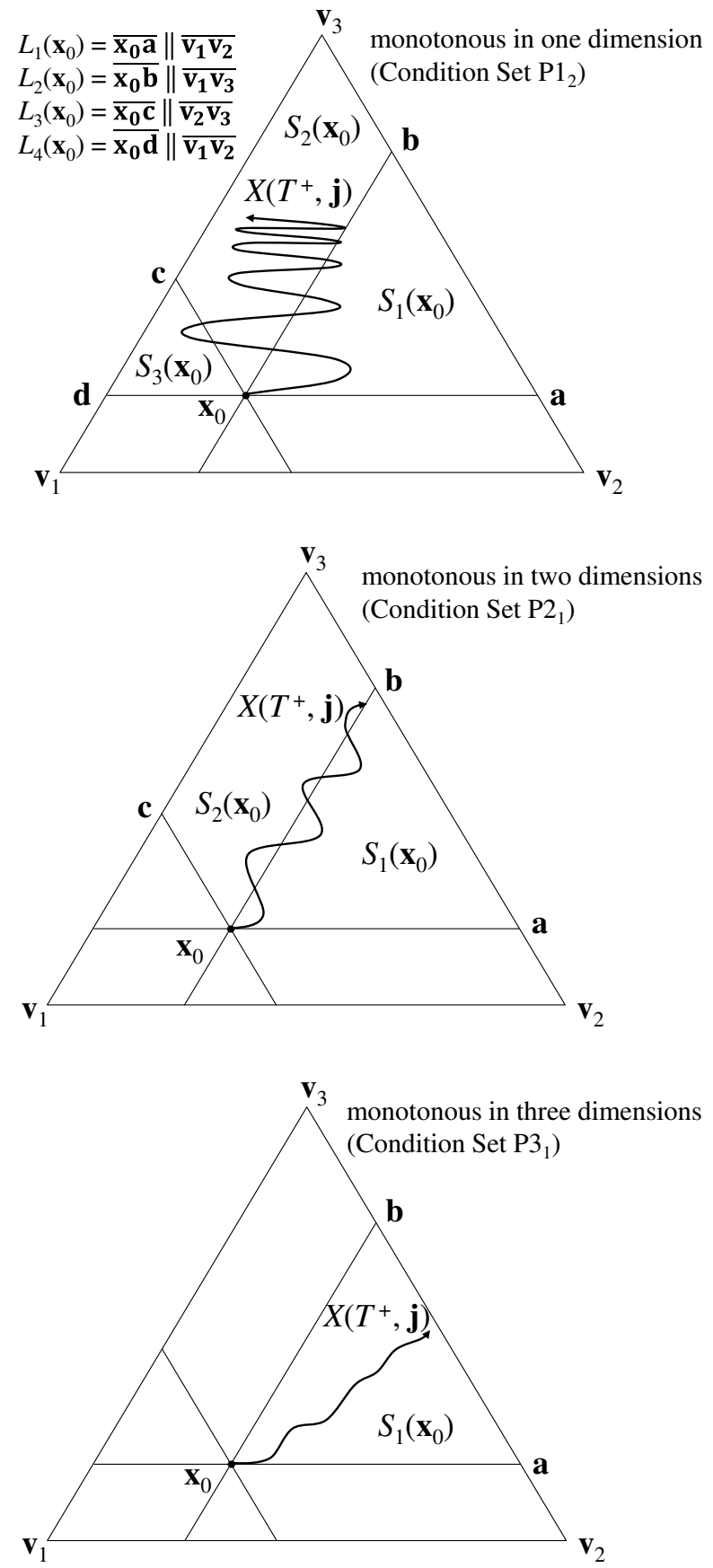

Figure 6. Examples of monotonous waves.
In the case of monotonicity in three dimensions, only the fixed point outcome is possible, as implied by the monotone convergence theorem: since each of the functions $x_{1}(t), x_{2}(t)$, and $x_{3}(t)$ is monotonous and restricted by an upper/lower limit of 0 and 1, each of the $x_{1}(t), x_{2}(t)$, and $x_{3}(t)$ converges to its fixed point $\left(x_{1}{ }^{*}\right.$, $x_{2}{ }^{*}$, and $x_{3}{ }^{*}$, respectively) or reaches it in finite time (and stays there). Thus, $\mathbf{x}(t)$ converges to a fixed point $\mathbf{x}^{*} \equiv\left(x_{1}{ }^{*}, x_{2}{ }^{*}, x_{3}{ }^{*}\right) \in S$ or reaches it in finite time.

Additionally, in the case of monotonicity in one dimension, the system may converge to a line segment if the trajectory is a wave. In this case, the wavelength decreases and the vector-angle range converges to the range of $180^{\circ}$ as the system converges to the line segment (see the first part of Figure 6).

Note that in the case of monotonicity in two dimensions, the convergence to a line segment is not possible, as explained in the following. If the trajectory converges to a line segment, the tangential vector angle range must increase to a range of $180^{\circ}$ which is prohibited by the definition of monotonicity in two dimensions, which allows only for a vector angle range of $120^{\circ}$. The former fact follows from the definition of the omega limit set, where for each of the points on the line segment (constituting the omega limit set), a sequence of points on the wave must be found that converges to it.

\section{Implications of Non-Self-Intersection for Transitional Dynamics}

While non-intersecting trajectories and limit dynamics are treated in Section 5, we focus, now, on the implications of (transversal) non-self-intersection for transitional dynamics. The class of transversally non-self-intersecting continuous trajectories on the 2 -simplex is a subclass of the class of continuous trajectories on the 2-simplex. Moreover, the class of non-self-intersecting continuous trajectories on the 2 -simplex is a subclass of the class of transversally non-self-intersecting trajectories on the 2-simplex, since the former does not allow for Jordan-curves in 
contrast to the latter. Thus, by imposing the condition of (transversal) non-self-intersection, we can reduce the set of feasible trajectories on the 2-simplex, which can be exploited in prediction of dynamics, as explained in the following.

Obviously, the non-self-intersection is an important constraint in systems of continuous trajectories on two-dimensional domains. In the case of discontinuous trajectories, non-self-intersection still may reduce the class of feasible trajectories significantly depending on the type of discontinuity and the physical/ social system being analyzed. However, in extreme cases and, in particular, in the case of point sequences on the 2-simplex (e.g., discrete-time paths) non-selfintersection becomes obsolete as a restraint (in natural and social sciences where the exact position of a system on the simplex is not measurable). For the same reasons, non-self-intersection is an obsolete restraint in three- or higher-dimensional dynamic system domains (see, e.g., Stijepic (2015)).

\subsection{Qualitative Simulation}

The (transversal) non-self-intersection constraint on continuous trajectories on the 2-simplex can be understood as a dynamic constraint: at each point of time $t \in T^{+}$, we have a restriction on system dynamics $\mathbf{x}(t, \mathbf{j})$ preventing certain type of dynamics (namely, the dynamics that correspond to a self-intersection of the trajectory). The constraint is dynamic in the sense that it changes over time. In particular, it depends on the current position of the system on the 2-simplex and the form of the trajectory segment $X\left(T^{-}, \mathbf{j}\right)$ representing the dynamics over the past time period $T^{-}$ (e.g., the longer the latter segment, the stronger is the constraint on the current dynamics), i.e., the constraint is updated continuously. This fact can be used in qualitative simulation, as discussed in detail by Lee and Kuipers (1988) and Kuipers (1986).

\subsection{Non-Self-Intersection in Combination with a Determined Trajectory-Segment}

The concept of (transversal) non-self-intersection can be very useful even if we do not assume the dynamic constraint view discussed in Section 4.1. In particular, assume that the trajectory segment $\left(X\left(T^{-}, \mathbf{j}\right)\right)$ representing past dynamics is given by empirical data on past dynamics or by empirical laws. Then, in general, $X\left(T^{-}, \mathbf{j}\right)$ can be used as a basis for a partitioning of the 2-simplex. For example, since the lines that are parallel to the 2-simplex edges have a clear intuitive interpretation, $X\left(T^{-}, \mathbf{j}\right)$ and such lines can constitute an intuitively meaningful partitioning of the simplex (see Stijepic (2015)). Then, paths on the 2 -simplex can be understood as sequences of partition switches, and the non-self-intersection constraint as an exclusion of certain switches, as demonstrated in Figure 7, where (immediate) switches between the partitions $A$ and $C$ are prohibited by the non-self-intersection constraint. If we interpret $t=0$ as present, $T^{-}$ as past, and $T^{+}$as future, this prohibition corresponds to infeasible future scenarios, i.e., non-self-intersection can be used in prediction of future dynamics (cf. Stijepic 2015).

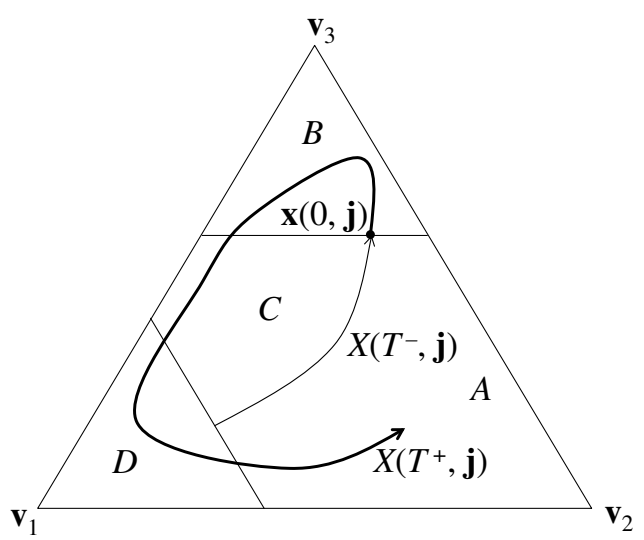

Figure 7. Paths as partition switches.

Obviously, depending on the (natural/social sciences) topic analyzed by these concepts, a certain length and positioning of $X\left(T^{-}, \mathbf{j}\right)$ may be necessary to derive significant predictions. In particular, if $X\left(T^{-}, \mathbf{j}\right)$ is rela- 
tively short or located in a relatively small or peripheral subset of the 2-simplex, it may not be possible to establish a relevant partitioning inducing a prohibition of paths that is of significant relevance for the topic/theory being analyzed (cf. Stijepic 2015). These requirements are well known in statistics, where the length of the past time series and avoidance of outliers is important for the (statistical) significance of the predictions based on empirical (time-series) data.

\section{Poincaré-Bendixson Theory}

The Poincaré-Bendixson theory, which is one of the fundaments of the dynamic systems theory, can be used to predict the qualitative properties of the limit dynamics of a smooth dynamic system in the plane. It applies to continuous systems, yet requires additional restrictions on the system (to ensure a sufficient degree of smoothness). We discuss here these requirements from a rather topological point of view applying the concepts discussed in Section 2.4. For a general, discussion of the requirements and predictions/statements of the Poincaré-Bendixson theory, see, e.g., Andronov et al. (1987, p.362f.), Ciesielski (2012), Guckenheimer and Holmes (1990, p.45), Hale (2009, p.55) and Teschl (2011, Chapter 7.3).

Assume that the dynamics on the 2-simplex are representable by a (relatively) smooth autonomous differential equation system in terms of the coordinates $\left(y_{1}, y_{2}\right)$ of a two-dimensional coordinate system that is parallel to the 2-simplex (see Figure 8). Then, the Poincare-Bendixson theory states that the limit dynamics of this system are either cyclical or transitory. In particular, the omega limit set of a trajectory generated by such a system consists of a fixed point, a Jordan curve, or a homo-/heteroclinic union (of curves and fixed points).

The geometrical interpretation of the requirement of the representability by a smooth differential equation system in $y_{1}-y_{2}$-coordinates is that the trajectories of the dynamic system on 2-simplex constitute a simple covering (of a connected subset) of the 2-simplex. In particular, such a simple covering consists of nonintersecting and transversally non-self-intersecting trajectories, where the union of these trajectories is a connected subset of the 2-simplex (see Stijepic 2017c for a detailed discussion and literature references).

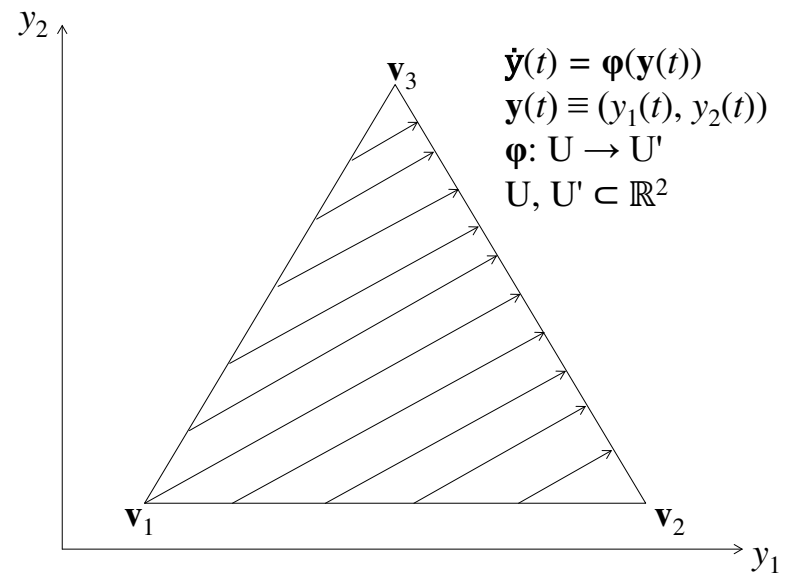

Figure 8. Representation of a dynamic system on $S$ by a two-dimensional differential equation system.

\section{Applications in Economics}

The mathematical theories of continuous dynamics on the standard 2-simplex developed in the previous sections have almost direct applications in the analysis of economic dynamics. In particular, they can be used in prediction of economic structural change, discussion of sectoral production functions, assessment of structural change costs, and design of cost-minimal structural change policies, as discussed in the following sections.

\subsection{Economic Topics Covered by the Models of} Continuous Dynamics on the 2-Simplex

A major pillar of economics is the study of long-run economic dynamics, where short-run fluctuations are neglected and the dynamic patterns that persist over long periods of time (e.g., 100 years) are studied. In this context, the concept of structural change is essential, where not only aggregate economic indices 
(e.g., gross domestic product, trade volume, and economy-wide employment) are studied but also their structure. In particular, the aggregate indices are subdivided into components and the significance of these components for the aggregate index is indicated by the components' shares in the aggregate index. Many of these 'shares', such as savings rate, investment rate, and sectoral employment shares, are well known even in public debates. In general, structural change refers to the dynamics of these 'shares', where the shares satisfy the conditions stated by (1). In other words, economic structural change, i.e., the long-run dynamics of the 'shares' can be depicted by trajectories on standard simplexes. Moreover, the assumption of continuous-time frameworks and continuous functional forms is a general convention in long-run economic dynamics modeling (although there are exceptions from this convention), which, in general, yields continuous dynamics of the shares on standard simplexes. For an overview of the topics that are covered by the system-theoretical models of continuous trajectories on standard simplexes and for corresponding references from the economics literature, see Stijepic (2017b).

To provide some details and references on the economic applications of the system-theoretical models derived in the previous sections, we focus on a specific sort of economic structural change, namely, long-run labor allocation dynamics in the three-sector framework, in Section 6.2.

\subsection{Example: Long-run Labor Allocation Dynamics}

The three-sector framework is one of the major concepts for studying economic structural change (for an overview of the literature, see, e.g., Schettkat and Yocarini (2006), Krüger (2008), Silva and Teixeira (2008), Stijepic (2011), Herrendorf et al. (2014), and Neuss (2018)). It is based on the assumption that economic activities can be divided into three categories or 'sectors': agriculture, manufacturing, and services. One of the major indices for studying the importance of these sectors are the shares of each of the sectors in aggregate employment (abbr. 'employment shares'). These shares and their dynamics can be depicted by continuous trajectories on the 2 -simplex (cf. Stijepic (2015, 2017a)). Thus, we can use the concepts discussed in Sections 2-5 in the analysis of labor allocation dynamics as discussed in the following.

While it is difficult to derive any consensus statements on the quantitative characteristics of labor allocation dynamics, the literature implies that there seem to be some empirically observable qualitative laws of labor allocation dynamics, which can be expressed by using the geometrical concepts discussed in our paper. In particular, Stijepic (2018) shows that the typical long-run labor allocation dynamics of a nowadays highly developed country over the last two centuries can be described by a trajectory that has the following characteristics: (1.) it is monotonous in two dimensions (cf. Sections 2.3 and 3) and (thus) non-self-intersecting (cf. Sections 2.4 and 4); (2.) it has a negative signed curvature $\underline{\kappa}$ (cf. Section 2.3); (3.) its initial segment is located in the simplex partition $S_{\mathrm{v} 1}$ (cf. (5) and Figure 2); and (4.) its final segment is located in partition $S_{\mathrm{v} 3}$ (cf. (5) and Figure 2). As discussed by Stijepic (2017a), these empirical observations can be interpreted as "natural" laws of structural change (since, among others, they are supported by the theoretical literature consensus) and, thus, can be exploited for predictions of structural change. For example, Proposition 2 and Corollary 1 (cf. Stijepic (2017a)) and the approach discussed in Section 4.2 (cf. Stijepic (2015)) can be used to predict the future (transitional) dynamics of labor allocation in developing and developed economies. Moreover, Stijepic (2017c) uses the empirical findings of Stijepic (2018) and the topological approach discussed in Section 5 for a discussion of the applicability of the Poincare-Bendixson theory in the prediction of limit dynamics of labor allocation. 
Beside these applications, which focus on prediction of structural change, the models of continuous dynamics discussed in our paper have further applications in in structural change modeling: Stijepic (2017d) shows that labor allocation trajectories that are monotonous in three dimensions minimize the structural change costs (e.g., unemployment, geographical relocation costs, and environmental pollution) and uses this result to elaborate a development policy minimizing the labor reallocation costs in a developing economy. Stijepic (2017e) uses the model of monotonous and continuous trajectories and the concept of curvature (cf. Section 2.3) to discuss a widespread assumption in theoretical structural change modeling (namely, the assumption of Cobb-Douglas production functions) by applying an axiomatic-geometrical approach. Finally, Stijepic (2017b) discusses how the topological concepts discussed in Section 2.4 can be interpreted and applied in the context of labor allocation dynamics modeling.

\section{Conclusions}

In this paper, we have studied models of continuous dynamics on the 2-simplex that arise when set-theoretical, differential-geometrical, or topological restrictions are imposed on the trajectories of the model. We focused on the qualitative properties of transitional and limit dynamics of these models and discussed their applications in long-run economic dynamics modeling.

Many of our results (in general, the results of Section 3 and their applications in economics) can be extended to discrete or discontinuous systems or higherdimensional simplexes. Yet the rather topological concepts discussed in Sections 4 and 5 (e.g., the Poincaré-Bendixson theory) are, in general, not applicable or not useful in discrete or higherdimensional systems and their applications (cf. Stijepic $2015,2017 b, c)$. In the latter systems, the concept of chaos as well as existence theorems on fixed points are of interest. Thus, further research could focus on them and, in particular, their system-theoretical significance for long-run economic dynamics.

Empirical evidence implies that there are fluctuations of the labor allocation shares that correspond to the waves on the 2-simplex discussed in Sections 3.2 and 3.3. Further research could focus on a detailed discussion of waves on the 2-simplex and the application of the resulting system-theoretical models in the explanation of the empirically observed waves arising in labor-allocation dynamics.

\section{Acknowledgment}

No funding has been received for this research.

\section{References}

[1] D. Stijepic, A geometrical approach to structural change modelling, Structural Change and Economic Dynamics 33 (2015) 71-85.

[2] D. Stijepic, Positivistic models of structural change, Journal of Economic Structures 6 (2017a) 1-30.

[3] D. Stijepic, Empirical evidence on the topological properties of structural paths and some notes on its theoretical explanation, MPRA Working Paper No. 82473, $2017 \mathrm{~b}$, available online at: https://mpra.ub.uni-muenchen.de/82473/1/MPRA_paper_ 82473.pdf

[4] W.W. Lee, B.J. Kuipers, Non-intersection of trajectories in qualitative phase space: a global constraint for qualitative simulation, Proceedings of the Seventh National Conference on Artificial Intelligence (AAAI-88), Morgan Kaufmann, Los Altos, CA, 1988.

[5] B. Kuipers, Qualitative simulation, Artificial Intelligence 29 (1986) 289-338.

[6] A.A. Andronov, A.A. Vitt, S.E. Khaikin, Theory of oscillators, Dover Publications, Mineola, New York, 1987.

[7] K. Ciesielski, The Poincaré-Bendixson theorem: from Poincaré to the XXIst century, Central European Journal of Mathematics 10 (2012) 2110-2128.

[8] J. Guckenheimer, P. Holmes, Nonlinear oscillations, dynamical systems, and bifurications of vector fields, Springer, New York, 1990.

[9] J.H. Hale, Ordinary differential equations, Dover Publications, Mineola, New York, 2009.

[10] G. Teschl, Ordinary differential equations and dynamical systems, American Mathematical Society, Providence, Rhode Island, 2012. 
[11] D. Stijepic, On the predictability of economic structural change by the Poincaré-Bendixson theory, 2017c, available at SSRN: https://ssrn.com/abstract=3015889.

[12] R. Schettkat, L. Yocarini, The shift to services employment: a review of the literature, Structural Change and Economic Dynamics 17 (2006) 127-147.

[13] J.J. Krüger, Productivity and structural change: a review of the literature, Journal of Economic Surveys 22 (2008) 330-363.

[14] E.G. Silva, A.A.C. Teixeira, Surveying structural change: seminal contributions and a bibliometric account, Structural Change and Economic Dynamics 19 (2008) 273-300.

[15] D. Stijepic, Structural Change and Economic Growth: Analysis within the Partially Balanced GrowthFramework, Südwestdeutscher Verlag für Hochschulschriften, Saarbruecken, 2011. An older version is available online: http://deposit.fernuni-hagen.de/2763/.
[16] B. Herrendorf, R. Rogerson, Á. Valentinyi, Growth and structural transformation, In: P. Aghion, S.N. Durlauf (Eds.), Handbook of Economic Growth, Volume 2B, Elsevier B.V., Amsterdam, 2014.

[17] L. van Neuss, The drivers of structural change, forthcoming in Journal of Economic Surveys 32 (2018).

[18] D. Stijepic, Empirical evidence on the geometrical properties of structural change trajectories, Research Journal of Economics 1 (2018): 1-10.

[19] D. Stijepic, On development paths minimizing the structural change costs in the three-sector framework and an application to structural policy, 2017d, available at SSRN: https://ssrn.com/abstract=2919806.

[20] D. Stijepic, An argument against Cobb-Douglas production functions (in multi-sector growth modeling), Economics Bulletin 37 (2017e) 1143-1150. 